\title{
Numerical modelling of 3D reconnection due to rotational footpoint motions
}

\author{
I. De Moortel ${ }^{1}$ and K. Galsgaard ${ }^{2}$ \\ ${ }^{1}$ School of Mathematics and Statistics, University of St Andrews, North Haugh, St Andrews, Fife KY16 9SS, Scotland \\ e-mail: ineke@mcs.st-and.ac.uk \\ 2 Niels Bohr Institute, Julie Maries vej 30, 2100 Copenhagen Ø, Denmark
}

Received 24 November 2005 / Accepted 30 January 2006

ABSTRACT

\begin{abstract}
The rapid dynamical evolution of the photospheric magnetic carpet provides a large energy source for the solar corona. In this context, the role of 3D magnetic reconnection is crucial in releasing the free magnetic energy, build up due to the continuous footpoint motions. To understand the processes by which this can take place, we have to obtain a better understanding of the basic reconnection process that can take place in 3D magnetic field configurations. In this paper, we investigate magnetic reconnection, driven by rotational footpoint motions, using 3D numerical MHD simulations. The model consists of two positive and two negative sources, which are placed symmetrically on opposite boundaries of the cubic domain. The initially potential fluxtubes are forced to interact by the rotational driving of the flux concentrations on the boundaries. We consider two variations of this setup, namely with and without an additional, constant, background magnetic field. In the no-background case, the magnetic connectivity is divided into independent regions by separatrix surfaces, while the case with a background field is represented by one global connectivity region. The dynamical evolution is followed and found to differ significantly from the comparable potential evolution. Strong currents are concentrated along separatrix surfaces or rapidly developing quasi-separatrix layers (QSLs). Investigating the reconnection rates of the systems shows that the stronger the background field is, the more efficient the reconnection process of the flux in the respective fluxtubes.
\end{abstract}

Key words. magnetohydrodynamics (MHD) - Sun: corona - Sun: activity - Sun: magnetic fields

\section{Introduction}

Many coronal heating mechanisms have been suggested which rely on magnetic reconnection to convert the energy stored in the solar magnetic field into heating the local plasma. Some of these mechanisms involve complex footpoint motions in the photosphere which slowly (compared to the local Alfvén speed) tangle and stress the coronal magnetic field. This braiding of footpoints causes a build up of magnetic energy and the formation of current concentrations, allowing reconnection to take place (Galsgaard \& Nordlund 1996; Gudiksen \& Nordlund 2005). In the Quiet Sun, the solar surface magnetic field is clustered in small flux concentrations, which are buffeted around by the granular motions and are continuously emerging, fragmenting, merging and cancelling (Schrijver \& Zwaan 2000; Parnell 2001, 2002; Hagenaar 2001). This magnetic carpet is continuously being replaced due to these processes and Hagenaar et al. (2003) estimated that the photospheric flux is recycled on a timescale of 8-19 h. Obviously, this dynamical behaviour of the photospheric flux sources has consequences for the coronal magnetic field. The complex connectivity structure of the solar magnetic field results in the presence of many separatrix surfaces, which divide regions of different connectivity (Close et al. 2004a). Following the original idea of footpoint braiding (Parker 1972; van Ballegooijen 1986; Mikić et al. 1987; Galsgaard \& Nordlund 1996), Priest et al. (2002) showed that even simple motions of any one of the photospheric magnetic fragments result in the rapid formation of current sheets along these separatrix surfaces, as well as separator (intersection of separatrix surfaces) current sheets. These large current concentrations will enable reconnection to occur and hence, the connectivity of the photospheric sources changes on a timescale much faster than the photospheric recycle time. Indeed, Close et al. (2004b), using a potential model, calculated that the coronal field is recycled in as little as $1.4 \mathrm{~h}$.

Although the individual contributions are likely to be small, the motions of the photospheric fragment will contribute significantly to the heating of the local coronal plasma. This constantly evolving magnetic carpet is likely to play an essential role in maintaining the $1 \mathrm{MK}$ background temperature of the Quiet Sun corona (see e.g. Parnell 2002; or Falconer et al. 2003). On the other hand, Pevtsov \& Acton (2001) present observational evidence suggesting that the high-temperature corona $(>2 \mathrm{MK})$ is primarily associated with the evolution of strong active region magnetic fields and large-scale reconnection. Many studies have focused on the energy release due to emergence or annihilation of flux fragments but far less attention has been paid to simple interactions of flux concentrations, which could be at least as important. However, several numerical simulations have recently modelled elementary heating events, driven by simple motions of the footpoints which do not result in a change in the total amount of flux (i.e. flux is not created or cancelled). For example, using the "minimum current corona" approach, Longcope (1998) investigated the interaction between flux sources in a constant background magnetic field and found that separator current sheets form. In a series of papers Galsgaard et al. (2000), Parnell \& Galsgaard (2004) and Galsgaard \& Parnell (2005) study similar interactions between two, initially unconnected flux fragments. When subjected to the imposed boundary driving, the flux systems are forced to interact, with the two sources connecting 
and subsequently disconnecting through separator reconnection (Haynes et al. 2006). By comparing the dynamical and potential evolution of the system, both reconnection processes are found to be fast. The amount of energy injected into the system by the boundary driving and the rate of Joule dissipation are found to be proportional to the distance travelled by the flux sources, not the speed at which they travel. Mellor et al. (2005) investigated the idea of fluxtube tectonics numerically, confirming that simple lateral motions of a non-uniform magnetic field results in the build up of current sheets. Using a reduced MHD model, analytical investigations by Priest et al. (2005) compare the energy storage and heating that occur at separators and separatrix surfaces by considering rotating and twisting of two adjacent flux concentrations and found that coronal heating is of comparable importance at separatrices and separators.

Both separatrix surfaces and separators are preferred locations for the build up of currents. However, these features require the existence of magnetic null points, i.e. locations where the magnetic field vanishes, or bald patches, i.e. locations where the magnetic field is tangential to the photospheric surfaces. When no nulls or bald patches are present, similar features can be defined, termed quasi-separatrix layers (QSLs) (Priest \& Démoulin 1995; Démoulin et al. 1996), where the change in magnetic fieldline connectivity is very large (rather than truely discontinuous). In the case where the magnetic flux patterns are not represented by flux sources on the boundary plane, the presence of many separatrix surfaces will be replaced by QSLs. Although these QSLs are known to be the sites of current formation (Milano et al. 1999), there is still much debate about the details of this process (Titov et al. 2003; Galsgaard et al. 2003; Mellor et al. 2005; Démoulin 2005; Aulanier et al. 2005). In particular, it appears that QSLs will have to be exposed to perturbations which initiate stagnation like motions, to rapidly attract current. Similar conditions seem to be valid for real separator lines (Galsgaard \& Nordlund 1997; Galsgaard et al. 2000).

In this paper, we present numerical simulations of one of the setups investigated by Priest et al. (2005), namely the reconnection that occurs when the footpoints of two adjacent fluxtubes are rotated. The basic model and the numerical code are described in Sect. 2 and a summary of both the potential and dynamical evolution is given in Sect. 3. Subsequently, the generation of current concentrations (Sect. 4), the connectivity structure and reconnection rates (Sect. 5) and the global energetics (Sect. 6) are investigated. A discussion of the results and the general conclusions can be found in Sects. 7 and 8, respectively.

\section{Numerical setup}

\subsection{Basic model}

The analytical model of Priest et al. (2005) considers two adjacent, initially straight, slender fluxtubes, which are subjected to rotational photospheric motions. We model this situation by considering two positive and two negative sources at the top and bottom of the cubical, numerical box, respectively (Fig. 1), given by

$B_{z}=B_{0}\left[\mathrm{e}^{-\left[\left(x-x_{1}\right)^{2}+\left(y-y_{1}\right)^{2}\right] / r_{0}^{2}}+\mathrm{e}^{-\left[\left(x-x_{2}\right)^{2}+\left(y-y_{2}\right)^{2}\right] / r_{0}^{2}}\right]+B_{\mathrm{bg}}$,

where $B_{0}=1$, the radius of the sources $r_{0}=0.065$ and $\left(x_{1}, y_{1}\right)=(0.3,0.5)$ and $\left(x_{2}, y_{2}\right)=(0.7,0.5)$ are the locations of the sources on both the top and bottom boundary. This implies that the sources are aligned on the top and bottom boundaries, resulting in two distinct, straight magnetic fluxtubes (Fig. 2 left). From the source distributions on the boundaries, a potential magnetic field configuration is found to fill the interior of the

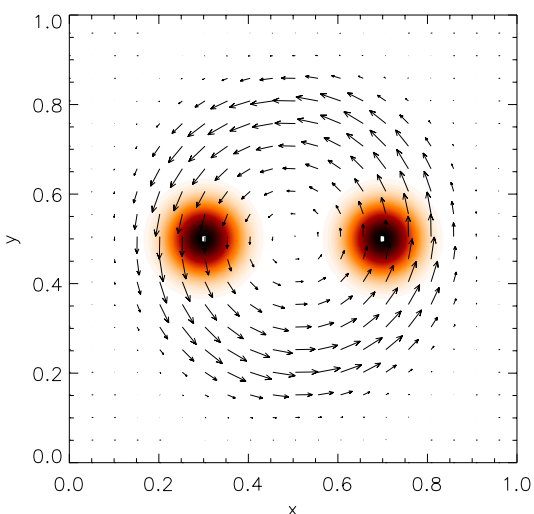

Fig. 1. Contour plot of the flux sources at the bottom boundary. The velocity vectors indicate the rotational driving imposed on the bottom boundary of the numerical domain.

data cube, assuming that the domain is periodic in the horizontal direction. We consider three different variations of this setup, namely with $B_{\mathrm{bg}}=(0,0.025,0.05)$. The flux sources are rotated at the boundaries, as shown in Fig. 1; going out from the centre of the box, the velocity increases linearly with radial distance, so that the flux concentrations maintain their original shape during the rotation. Further out, the velocity is reduced sharply, to minimise the rotational shear on the edges of the computational box. The velocity profile is given by

$v(r)=v_{0} r[1+\tanh (A(1-B r))]$,

where $r^{2}=x^{2}+y^{2}, v_{0}=0.02222$ and where $A=8.4$ and $B=1.4$ determine the steepness and location of the drop off in $v$ near the boundary. To accelerate the effect of rotation, the sources at the top and bottom boundary are counter-rotated, i.e. the (anticlockwise) velocity vectors shown in Fig. 1 represent the rotation on the bottom boundary. The driver on the top boundary has exactly the same profile, but with the opposite sign, i.e. clockwise. To ease the comparison between both variations of the setup, we use the angle $\theta$ by which the centre of the flux sources on each of the boundaries have been rotated as a reference value. Note that, as the two boundaries are counter-rotated, the fluxtubes connecting both boundaries are subject to a "real" rotational angle of $2 \theta$. To simplify the discussion below, we will occasionally refer to the sources on the bottom boundary as $B_{1}$ and $B_{2}$ and to those on the top boundary as $T_{1}$ and $T_{2}$.

As the sources on the top and bottom boundary are aligned, the initial structure consists of two adjacent, vertical fluxtubes connecting the sources ( $B_{1}$ to $T_{1}$ and $B_{2}$ to $T_{2}$ ) when no background magnetic field is present and hence, the computational box contains two independent flux domains (Fig. 2 - top left), separated by a separatrix surface. Additionally, the imposed horizontal periodicity of the domain introduces separatrix surfaces on the domain boundaries. When a positive background field is added, there are no clear, distinct flux domains, as the whole boundary is penetrated with magnetic flux of the same polarity. However, for practical purposes, we can define flux associated with the sources as the flux within a radius $r_{0}$ (the radius of the sources in the no-background field case) of the centre of each of the sources. We can think of the volume enclosed by the fieldlines traced from the "edge", $r_{0}$, of each source as the core of the fluxtube and to ease the notation and discussion below, we will refer to them simply as "fluxtubes". Note that when a positive background magnetic field is present, the fluxtubes are not real topological features. They are merely a convenient notation to 

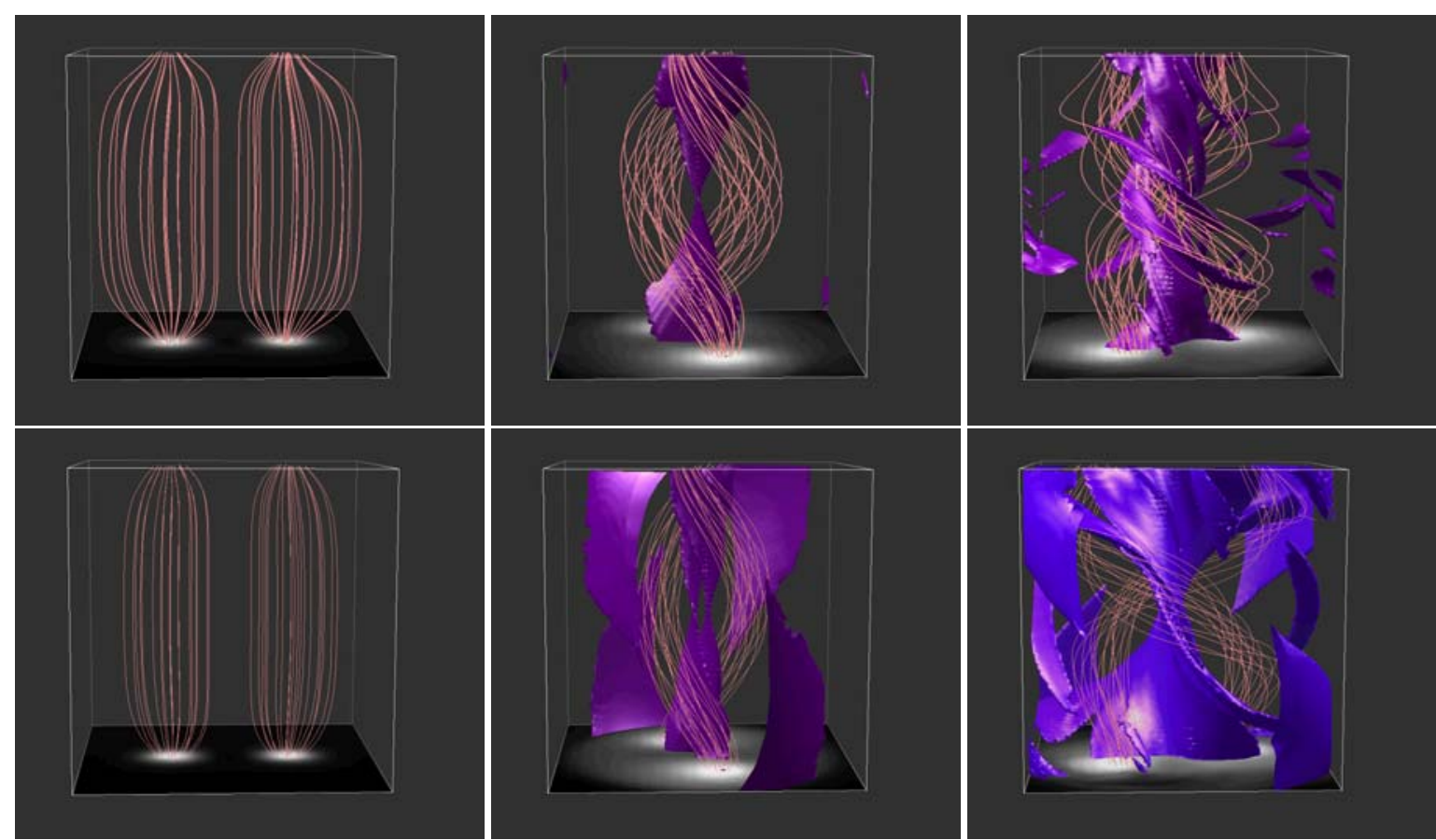

Fig. 2. Fieldlines traced from the flux concentrations on the bottom boundaries at a rotational angle $($ left $) \theta=0,($ middle $) \theta \approx 2$ and $($ right $) \theta \approx 3.75$ for $B_{\mathrm{bg}}=0$ (top) and $B_{\mathrm{bg}}=0.05$ (bottom). The isosurfaces represent current.

refer to flux associated with each of the sources on the boundaries. Using this concept, we can now define two flux domains and a background domain. In this context, the background domain keeps the fluxtubes apart at the start of the experiment, unlike in the no-background case, where the two (real) fluxtubes are in contact.

\subsection{Numerical code}

To follow the dynamical evolution of the 3D system, the numerical code solves the non-ideal MHD equations given by,

$$
\begin{aligned}
& \frac{\partial \rho}{\partial t}=-\nabla . \rho \boldsymbol{v}, \\
& \frac{\partial \rho \boldsymbol{v}}{\partial t}=-\nabla \cdot(\rho \boldsymbol{v} \boldsymbol{v}+\underline{\tau})-\nabla p+\boldsymbol{j} \times \boldsymbol{B}, \\
& \frac{\partial e}{\partial t}=-\nabla .(e \boldsymbol{v})-p \nabla \cdot \boldsymbol{v}+Q_{\text {joule }}+Q_{\mathrm{visc}}, \\
& \frac{\partial \boldsymbol{B}}{\partial t}=-\nabla \times \boldsymbol{E}, \\
& \boldsymbol{E}=-(\boldsymbol{v} \times \boldsymbol{B})+\eta \boldsymbol{j}, \\
& \boldsymbol{j}=\nabla \times \boldsymbol{B},
\end{aligned}
$$

where $\rho$ is the density, $\boldsymbol{v}$ the velocity, $\underline{\tau}$ the viscous stress tensor, $p$ the gas pressure, $\boldsymbol{j}$ the electric current, $\boldsymbol{B}$ the magnetic field, $e$ the thermal energy, $Q_{\text {joule }}$ and $Q_{\text {visc }}$ the joule and viscous dissipation, respectively and $\boldsymbol{E}$ is the electric field. The equations are solved in a 3D, Cartesian box, which is assumed to be periodic at the sides, and bounded in the vertical direction (i.e. the direction perpendicular to the plane in which driver is situated). Note that the MHD equations have been non-dimensionalised and dimensional quantities can be obtained by choosing three characteristic values and then using the following scaling relations:

$$
\begin{aligned}
& v_{0}=l_{0} / t_{0}, \\
& e_{0}=\rho_{0} v_{0}^{2},
\end{aligned}
$$

$$
\begin{aligned}
B_{0} & =v_{0} \sqrt{\mu_{0} \rho_{0}}, \\
T_{0} & =\tilde{\mu} v_{0}^{2} / R, \\
E_{0} & =v_{0} B_{0}, \\
j_{0} & =B_{0} /\left(\mu_{0} l_{0}\right),
\end{aligned}
$$

where the magnetic permeability $\mu_{0}=4 \pi \times 10^{-7} \mathrm{H} \mathrm{m}^{-1}, \tilde{\mu}=$ 0.6 and the gas constant $R=8.3 \times 10^{3} \mathrm{~m}^{2} \mathrm{~s}^{-2} \mathrm{~K}^{-1}$. For a more detailed and comprehensive description of the numerical code, see Nordlund \& Galsgaard (1997).

\section{Dynamical evolution}

As the boundary driving of the flux sources starts, the flux domains are forced to interact. For comparison, both the potential and dynamical evolution are calculated at each time step for the different values of $B_{\mathrm{bg}}$. The evolution of the potential field with $B_{\mathrm{bg}}=0$ is relatively straight forward: whenever the rotational angle is a multiple of $\pi$, the sources are realigned and hence, the initial configuration is recovered, with all the flux from the bottom sources $B_{1}$ and $B_{2}$ connecting to $T_{1}$ and $T_{2}$, respectively. At odd multiples of $\pi / 2$, the sources are "counter-aligned", i.e. all the flux from source $B_{1}$ is connected to $T_{2}$ and vice versa. At intermediate angles, the flux is divided between the sources, according to their relative positions. For example, at $\theta \approx \pi / 4$, half of the flux of $B_{1}$ will be connected to $T_{1}$, and the other half to $T_{2}$. The same is of course true for the flux associated with $B_{2}$. When a constant, weak background field is present $\left(B_{\mathrm{bg}} \neq 0\right)$, the configuration at intermediate rotation angles differs from the $B_{\mathrm{bg}}=0$ case: some of the flux from the sources now connects to parts of the (opposite) boundary associated with the background field, as the potential field represents the lowest energy state. In this case, the $\theta=\pi / 4$ state represents the Hyperbolic fluxtube setup used by Titov et al. (2003) to investigate the current growth at a QSL.

We now turn our attention to the dynamical evolution and three different stages, both for the case without (top) and 

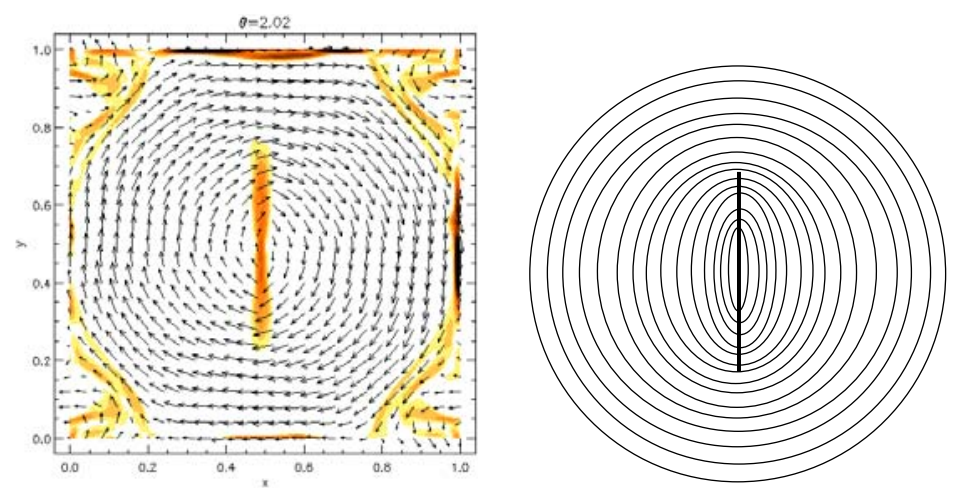

Fig. 3. (left) Contour of the current at $z=0.5$ and $\theta \approx 2$ for $B_{\mathrm{bg}}=0$. Overplotted are the magnetic field vectors in the plane $z=0.5$. (right) Sketch of the projected fieldlines structure and the separator current sheet.

with (bottom) background magnetic field, are shown in Fig. 2. Fieldlines traced from the flux sources on the bottom boundary, as well as isosurfaces of current are shown for a rotational angle of (left) $\theta=0$, (middle) $\theta \approx 2$ and (right) $\theta \approx 3.75$. It is immediately clear that the dynamical evolution bears little resemblance to the potential evolution described above. The images show that the magnetic fieldlines connecting the flux concentrations on both boundaries become increasingly twisted and stretched and that a twisted current sheet builds up along the (quasi-) separatrix surfaces. When a background magnetic field is added, the two fluxtubes are more confined and initially not in contact as they are separated by this background field. As the sources rotate, the fluxtubes approach each other and eventually collide. The rotational driving causes the two fluxtubes to twist around one another. The tension in the twisted fluxtubes initiates a stagnation flow that builds up a current sheet, through which reconnection eventually takes place and the connectivity of the fieldlines gradually changes. Two new flux domains are created in both sets of experiments, and a QSL forms at the interface between the two fluxtubes in the $B_{\mathrm{bg}} \neq 0$ case.

Let us look at the structure of the fieldlines in some more detail. Figure 2 shows that initially, i.e. at $\theta=0$, the field expands rapidly above the sources and becomes essentially uniform away from the boundaries. As the rotational driving gets established throughout the computational domain, the initially straight fieldlines connecting the top and bottom sources take on a helical shape. Projected onto e.g. the midplane of the domain $(z=0.5)$, the magnetic field structure in the plane looks almost circular, wrapping around an O-point. Figure 3 (left) shows a contour plot of the current sheet at $z=0.5$ for $\theta \approx 2$, as well as the magnetic field vectors in this plane. We see that, due to the continuous rotational boundary driving, the circular structure developed in the early phase of the experiment gets distorted, with the central O-point becoming increasingly elongated and with a separator current sheet lying along the central separatrix surface. The evolution of this central current sheet will be discussed in more detail in Sect. 4. The collapse phase of the O-point into a current sheet is driven by the increasing Lorentz stress, which the twisted fieldlines exert on the plasma in between the two original fluxtubes. As the location of the strong fluxtubes changes with distance from the driving boundaries, the orientation of the central current sheet twists along this axis. Note that the fieldline and current structure in the $z=0.5$ plane corresponds to the analytically predicted structure of Priest et al. (2005), a replica of which is shown in Fig. 3 (right) (or see the original in their Fig. 10). The tension force in the twisted fieldlines sets up a stagnation point flow centered on the O-point, carrying fieldlines
Table 1. An overview of the rotational angles at which various stages of the reconnection occur, for different values of the additional background magnetic field, $B_{\mathrm{bg}}$.

\begin{tabular}{lccc}
\hline \hline & $B_{\mathrm{bg}}=0$ & $B_{\mathrm{bg}}=0.025$ & $B_{\mathrm{bg}}=0.05$ \\
\hline Onset of reconnection & 2.04 & 2.17 & 2.42 \\
$25 \%$ reconnected flux & 3.27 & 2.98 & 3.05 \\
$50 \%$ reconnected flux & 3.83 & 3.42 & 3.30 \\
$75 \%$ reconnected flux & 4.30 & 3.74 & 3.49 \\
$100 \%$ reconnected flux & - & - & 3.74 \\
End of experiment & 4.78 & 3.86 & 4.08 \\
\hline
\end{tabular}

towards the current sheet. The increasing elongation of the sheet allows oppositely directed fieldlines to approach each other and hence, reconnection can take place, all along the current sheet. A summary of the different stages of reconnection (in terms of the amount of reconnected flux as a percentage of the total flux of a given source) is given in Table 1, for different strengths of the background magnetic field, $B_{\mathrm{bg}}$. The onset of reconnection between the fluxtubes takes place at increasingly large rotational angles as the magnitude of $B_{\mathrm{bg}}$ increases, due to its confining effect on the fluxtubes. However, comparing the different values of $\theta$ shows that, once started, the reconnection processes becomes increasingly faster with larger $B_{\text {bg }}$ values. We will investigate the reconnection rates in more detail in Sect. 5 .

Finally, a good indicator of reconnection is the parallel (i.e. along the magnetic fieldlines) component of the electric field (Linton \& Priest 2003; Priest et al. 2003; Pontin et al. 2005; Hesse et al. 2005). The fieldlines traced in Fig. 4 from one of the sources are coloured according to the magnitude of the parallel electric field $\left(E_{\mathrm{par}}\right)$. We can see that the innermost fieldlines, i.e. those fieldlines closest to the twisted current sheet, have the highest value of $E_{\mathrm{par}}$ (coloured red) and these fieldlines have reconnected to the other flux concentration in subsequent snapshots. Note that these fieldlines are only touching the current sheet at one central location. As the structure of the $E_{\mathrm{par}}$ region aligns with the current sheet, then different fieldlines are reconnecting all along the current sheet at the same time.

\section{Current evolution}

In this section, we investigate the qualitative evolution of the currents in the mid-plane of the computational domain $(z=0.5)$. Figure 5 shows the current structure at mid-height in the box, both for $B_{\mathrm{bg}}=0$ (top) and $B_{\mathrm{bg}}=0.05$ (bottom), where lighter colours correspond to higher values. Going from left to right, snapshots at increasing rotational angles $\theta$ are shown. 

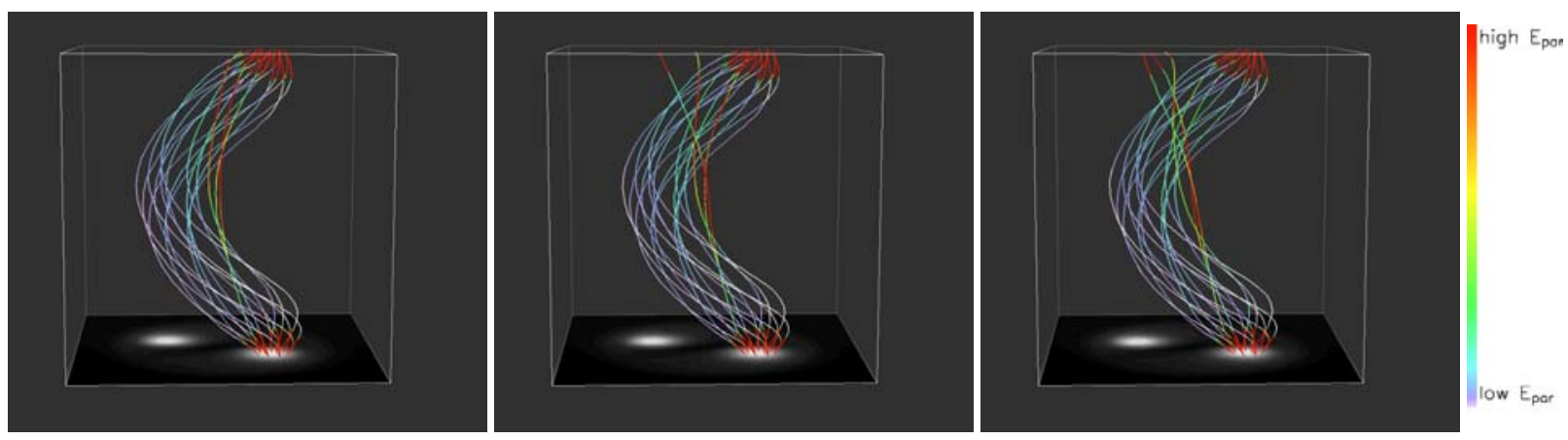

Fig. 4. Fieldlines traced from one of the bottom boundary flux concentrations and coloured according to the value of the local parallel electric field, with $B_{\mathrm{bg}}=0.05$, at a rotational angle $\theta=2.70$ (left), $\theta=2.76$ (middle) and $\theta=2.80$ (right).

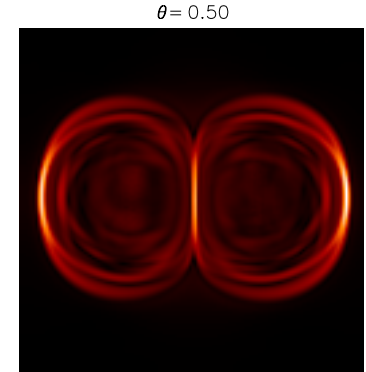

$\theta=0.49$

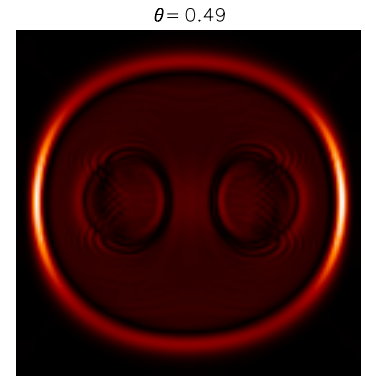

$\theta=2.04$

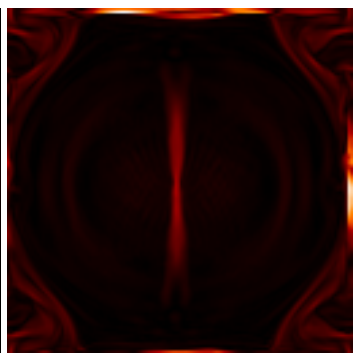

$\theta=2.02$

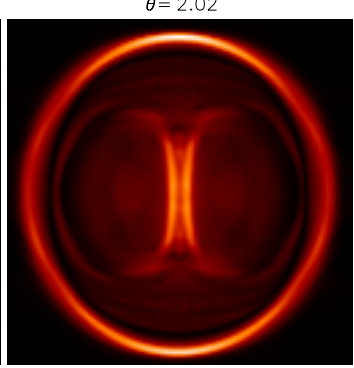

$\theta=2.49$
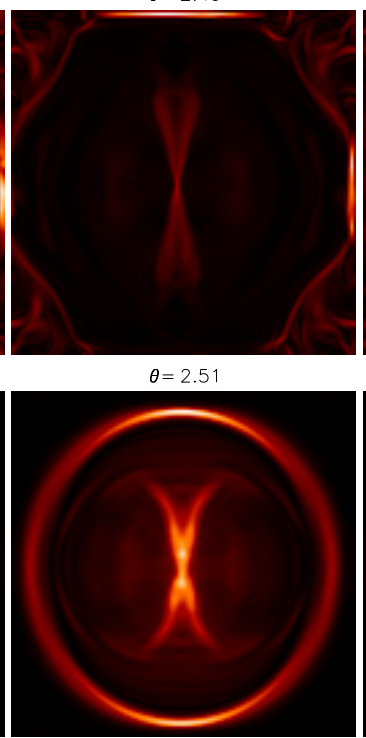

$\theta=3.02$

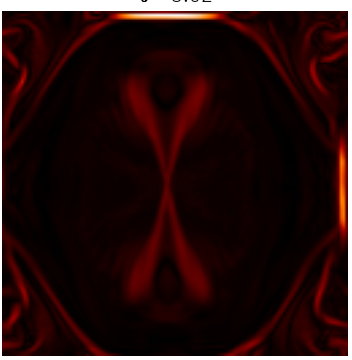

$\theta=3.02$

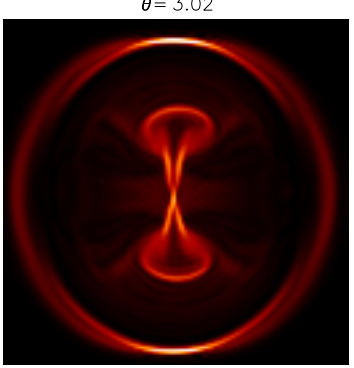

$\theta=3.77$

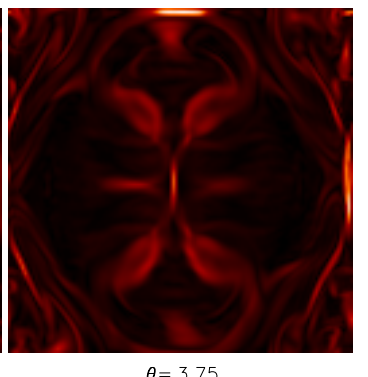

$\theta=3.75$

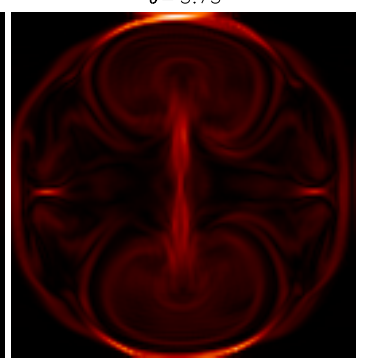

Fig. 5. Contour plots of the current at $z=0.5$ at various different rotational angles, for the case without (top) and with (bottom) background field, $B_{\text {bg }}=0.05$.

When $B_{\mathrm{bg}}=0$, some current builds up on the edges of the fluxtubes as the rotational driving starts, due to the shear caused by the relatively steep drop off in the amplitude of the driving velocity near the outer edges of the domain (see Fig. 1). These current structures show that the fluxtubes are initially indeed in contact at the centre of the domain. As the rotational driving continues, the central current sheet, lying along the separatrix surface, becomes increasingly strong, eventually allowing reconnection to occur (at $\theta=2.04$ ). Two new flux domains are subsequently created and the current along the separatrix surfaces bifurcates into an $x$-type configuration, with a separator along the intersection. At later stages, the current distribution evolves into a complex structure. This is also evident from Fig. 2 (top row - right), which shows not only the central twisted current sheet but also an additional helical current structure, wrapping around the central sheet. Finally, note that in the absence of a confining background field, some of the magnetic field will be almost purely horizontal close to the source boundaries and hence, the rotational driving will cause relatively large currents to build up along the periodic interfaces of the domain, as is evident in some of the graphs in Fig. 5 (top row).

The bottom row in Fig. 5 shows the corresponding current structures for the $B_{\mathrm{bg}}=0.05$ case. Again we can see some current along the edges of the fluxtubes, which are now not in contact at the start of the experiment. When a background field is added, some of the field in the mid-plane is not connected to the flux sources on the boundaries and hence, the flux anchored outside the driving profile will experience no rotation. The shearing at the interface between "rotated" and "non-rotated" field therefore results in the circular current structure, which is clearly evident in all the images of Fig. 5 (bottom row). The background magnetic field, situated between the two fluxtubes, is also subject to the rotational driving and hence, as the central current sheet builds up, this background field will start to reconnect. This process will remove the flux between the fluxtubes, allowing them to approach the central current sheet. When the edges of the fluxtubes meet in the centre of the domain, reconnection between the fluxtubes will start (at $\theta=2.42$ for $B_{\mathrm{bg}}=0.05$ ) and a similar x-type current structure as was seen for $B_{\mathrm{bg}}=0 \mathrm{de}$ velops. However, the magnitude of the current in the mid-plane in the $B_{\mathrm{bg}}=0.05$ reaches values almost double of those obtained when no background field is present. Finally, we point out the "bowshaped" structures that are present in the background case, at the later stages of the experiment, which result from the reconnection jets. As the sources are rotated further, these bowshaped currents propagate outward from the central region with sub sonic and sub Alfvénic velocites, until they eventually collide with the circular current at the edge of the domain. 

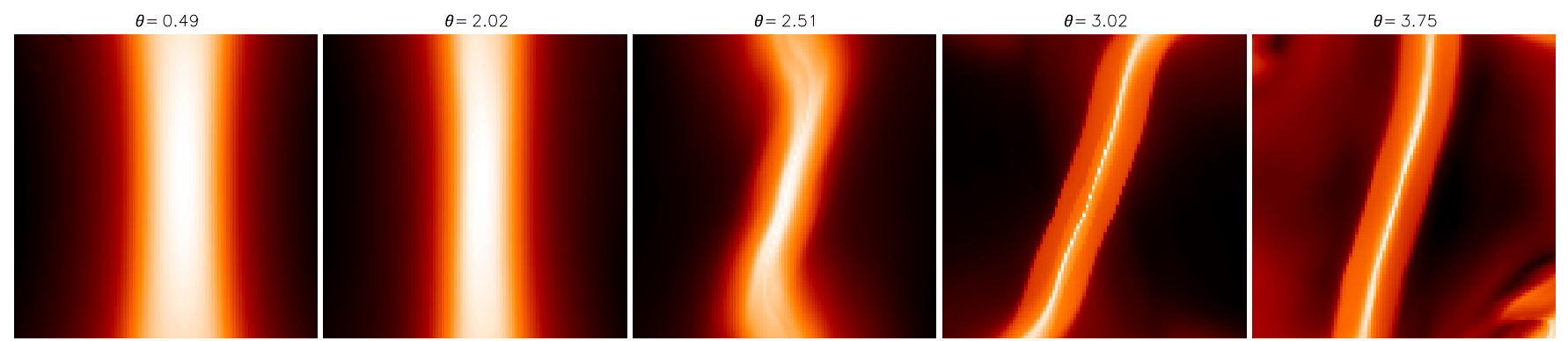

Fig. 6. Contour plots of the value of the squashing degree $Q$ at $z=0.5$ (for the central square of width 0.4 ), for the case with background field, $B_{\mathrm{bg}}=0.05$, at various different rotational angles.

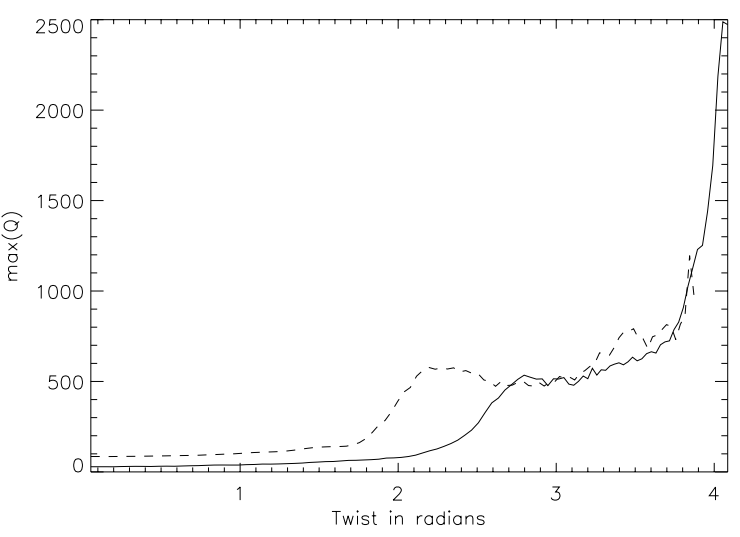

Fig. 7. The maximum value of the squashing factor $Q$ in the central square of width 0.4 , at $z=0.5$ as a function of the rotational angle. The solid and dashed lines correspond to $B_{\mathrm{bg}}=0.05$ and $B_{\mathrm{bg}}=0.025$, respectively.

The nature of the bowshaped and $\mathrm{x}$-type current structures will be investigated in more detail below but first, we look at the location of the current build up, when a background magnetic field is present.

As pointed out above, there is no real separation of connectivity domains when $B_{\mathrm{bg}} \neq 0$. However, it was noted by Priest \& Démoulin (1995) that the condition of a discontinuous jump in fieldline connectivity can be weakened to a local "large" spatial variation in the connectivity. Fluxtubes which such a large variation are called quasi-separatrix layers (QSLs) and can be detected by measuring the degree of squashing, $Q$, where QSLs are defined as fluxtubes with large values $(\gg 2)$ of $Q$ (see Titov et al. (2002) for a detailed definition). The degree of squashing was determined for the central square of width 0.4 , at the midplane $(z=0.5)$, and different snapshots are shown in Fig. 6, for the case $B_{\mathrm{bg}}=0.05$. Note that the scaling across the various snapshots is not comparable as they have not been normalised to a common value. Nevertheless, it is clear that a strong quasiseparatrix layer builds up in the mid-plane and evolves as the sources are rotated. In Fig. 7 the maximum value attained by the squashing factor $Q$ in the (reduced) central plane has been plotted as a function of the rotation angle. The solid and dashed lines correspond to $B_{\mathrm{bg}}=0.05$ and $B_{\mathrm{bg}}=0.025$, respectively. Note that, even at $\theta=0$, the value of $Q$ is already much larger than two, providing some justification for our concept of "quasidomains" in Sect. 3. For both values of the background field, the value of $Q$ changes only slightly in the early stages of the experiment. The fact that $Q$ is larger for weaker background field, $B_{\mathrm{bg}}=0.025$, is due to the fact that this provides a larger contrast between the flux sources and the background field, and hence, a somewhat steeper change in connectivity. Near the onset of reconnection between the fluxtubes, which occurs at larger $\theta$ for the stronger $B_{\mathrm{bg}}$, the maximum value of $Q$ builds up substantially, reaching similar values for both cases. The dramatic rise in $Q$ in the final stages of the $B_{\mathrm{bg}}=0.05$ experiment is due to the rapid opening of the fieldlines, as they almost all reconnect to the neighbouring domain (i.e. across the periodic boundaries of the domain). Finally, a comparison of Fig. 5 (bottom row) and Fig. 6 confirms that the currents do indeed build up along the QSL, similar to the build up of currents along the "real" separatrix surfaces in the no-background case. The reason only one "leg" of the x-type configuration is visible in the $Q$-values (Fig. 6) is that the tracing of the fieldlines, on which the determination of $Q$ is based, is only done in one direction, namely from the mid-plane to the top boundary of the numerical domain. Tracing in the other direction, i.e. from the mid-plane to the bottom boundary, will complete the x-type structure.

In this last part of our study of the currents, we look in more detail at the nature of the bowshaped and x-type structures which can be seen in Fig. 5. We again concentrate on the mid-plane, $z=0.5$, in the numerical box and Fig. 8 shows the contours of the current at $\theta \approx 3$, with cotemporal velocity (left) and magnetic field (right) vectors overplotted, both for $B_{\mathrm{bg}}=0$ (top) and $B_{\text {bg }}=0.05$ (bottom). At this stage in the evolution, the current sheet along the (quasi-)separatrix surfaces has developed into an obvious x-type configuration, and the bowshaped structures are also clearly visible (for $B_{\mathrm{bg}}=0.05$ ). For both cases, there is a stagnation point flow, carrying material into the reconnection region and the outflow from the reconnection region is also evident. The inflow is generated by the growing fieldline tension in the twisted fieldlines, while the outflow is accelerated by the tension in the reconnected fieldlines. Note how the outflow is not only directly out from the central reconnection region (perpendicular to the inflow) but is also channeled along the (quasi)separatrix layers. Looking at the cotemporal magnetic field vectors, we can see that the central O-point has become elongated and that there is a rapid shear in the magnetic field vectors going across the legs of the x-configuration. Although the contours in Fig. 8 represent the amplitude of the current, looking at the individual components of the current shows that the dominant contribution comes from the $z$-component, caused by this shear in the magnetic field component $B_{y}$, as $j_{z} \sim \partial B_{y} / \partial x$.

At first glance, an X-type current structure, a stagnation point flow and an O-point magnetic field configuration might appear contradictory. However, one has to bear in mind that Fig. 8 is a 2D projection of a 3D configuration. Figure 9 shows two different viewpoints (left - sideview; right - topview) of fieldlines passing through the 4 different regions across the central x-configuration: two fieldlines (yellow \& red) are approaching the central reconnection region, whereas the other two (blue \& 

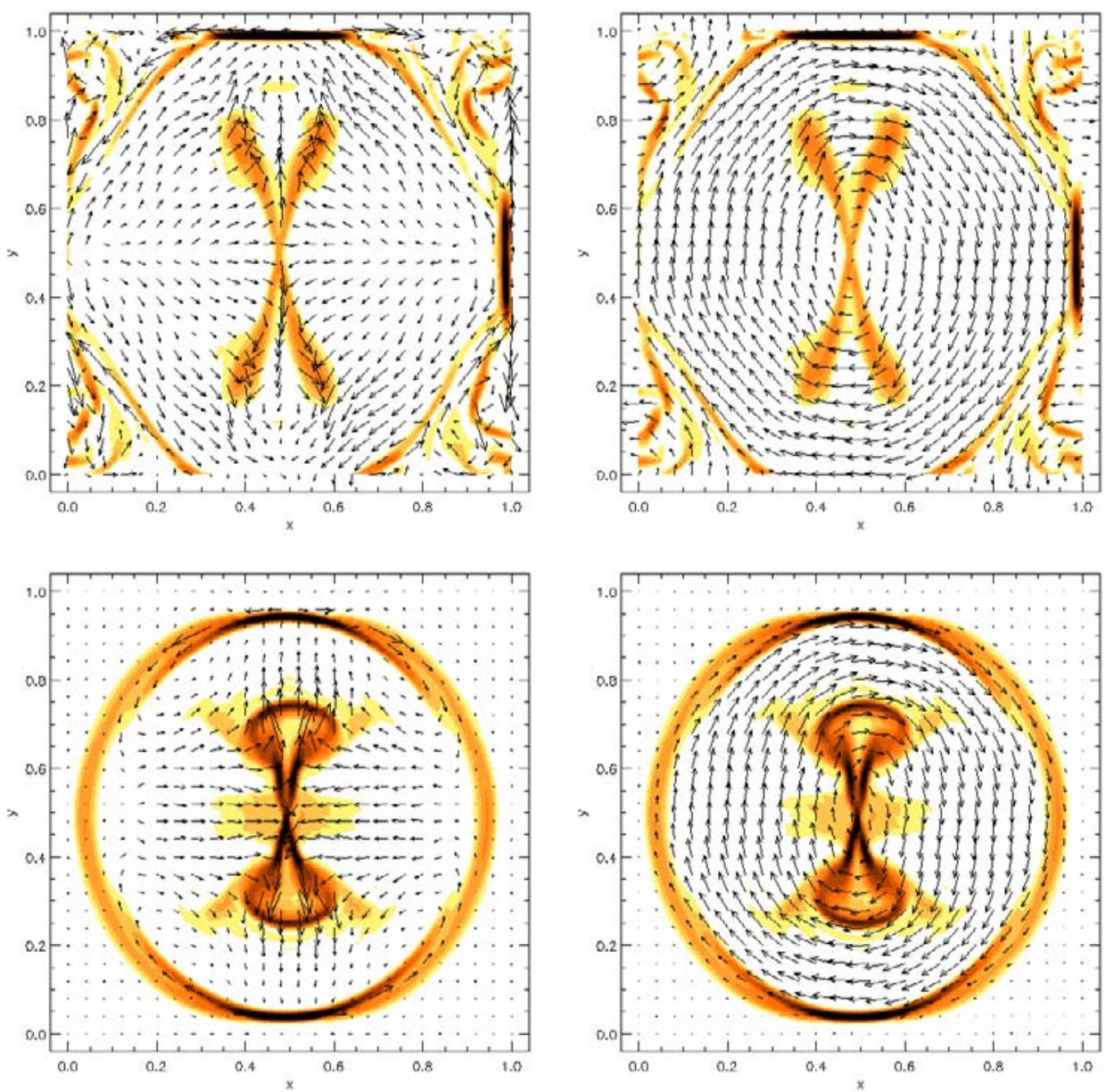

Fig. 8. Contour of the current at $z=0.5$ and $\theta \approx 3$ for the case without (top) and with (bottom) background field $\left(B_{\mathrm{bg}}=0.05\right)$. Overplotted are the cotemporal velocity (left) and magnetic field (right) vectors in the $z=0.5$ plane.
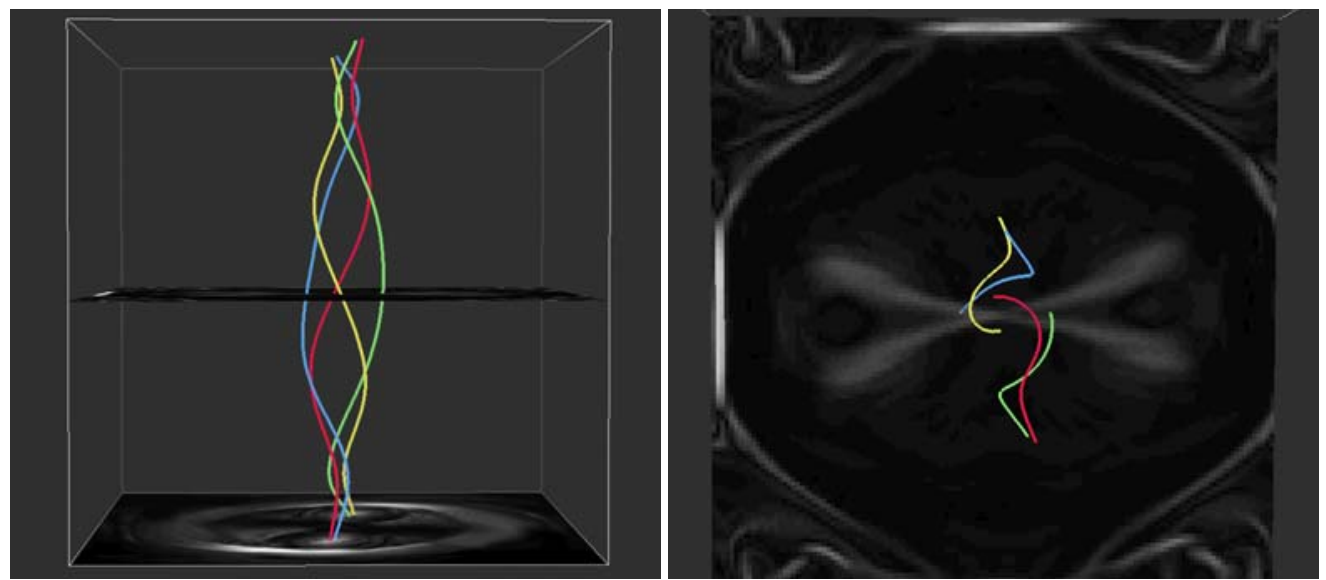

Fig. 9. Side on view and top view of four fieldlines around the central reconnection region at $\theta \approx 3$. Contours of current in the $z=0$ and $z=0.5$ planes are also shown.

green) are just leaving it for the $B_{\mathrm{bg}}=0$ case. If one compares the two graphs carefully, one can see that fieldlines coming from the same source on the bottom boundary connect to different locations on the top boundary, depending on whether they are approaching or leaving the reconnection region. The connections of the fieldlines in the inflow region (red \& yellow) are the ones that are originally present in the system (i.e. $B_{1}$ to $T_{1}$ and $B_{2}$ to $T_{2}$ ). As this type of fieldlines reconnect they will move into the left and right side of the $X$ (blue \& green), which now connect $B_{1}$ to $T_{2}$ and vice versa. Note also that, as expected, the reconnected fieldlines are much "straighter" (i.e. contain less twist) than the original, reconnecting ones. The topview (Fig. 9 - right) shows that the projection onto the midplane of both the reconnecting fieldlines (red \& yellow) and the reconnected fieldlines (blue \& green) will result in an O-point configuration, as shown in Fig. 8 (top right), rather than the classical reconnection 


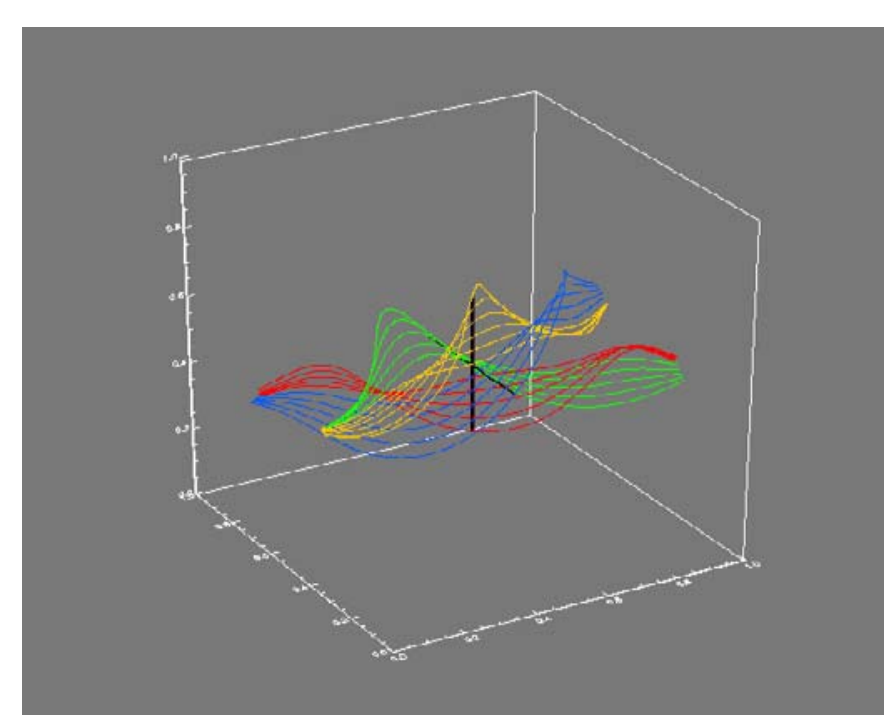

Fig. 10. The fieldline connectivity of the $B_{\mathrm{bg}}=0$ case at $\theta \approx 3$. The fieldlines are traced from the two black lines forming a cross at the center of the domain. The yellow and red fieldlines represents the original connectivity, while the blue and green fieldlines have reconnected.

$\mathrm{x}$-configuration. The kink that is apparent in the topview of the fieldlines, away from the central region, is the projected view of the rapid expansion of the concentrated flux sources near the top (and bottom) boundary of the domain. Finally, Fig. 10 shows a series of fieldlines traced from starting points along straight lines (thick black lines) across the inflow (red \& yellow fieldlines) and outflow (blue \& green fieldlines) regions. This picture confirms the presence of different connectivity domains going across the central reconnection region,

In the above paragraph, we showed how the currents build up along the (quasi-)separatrices. To investigate the nature of these currents further, we look at cross-sections of various variables across the current structures. Figure 11 shows cross-sections of current (thick solid line), vorticity (thin solid line), total pressure (dot-dot-dashed line) and $v_{x}$ (thin dot-dashed line) and $v_{y}$ (thin dashed line), as well as $B_{x}$ (thick dot-dashed line) and $B_{y}$ (thick dashed line). The horizontal, dashed lines outline the respective zero levels for the velocity and magnetic field components. The different quantities have been scaled in arbitrary ways to ease the (qualitative) comparison, looking for shock structures. Let us start with the nature of the x-type current. To examine the nature of this current properly, we need a cross-section perpendicularly across the structure which is why the cross-sections (along constant $y=0.6$ ) in the left-hand graph in Fig. 11 are taken at a height of $z=0.4$. At this height, the twisted central current sheet is orientated in such a way, that one of the legs of the x-type current is parallel to the $y$-axis and hence, cross-sections at constant $y$ will be normal to this particular part of the current structure. The vertical dotted lines outline the position of the leg of the $\mathrm{x}$ structure that is parallel to the $y$-axis. The peak in current just to the left hand side of the dotted line indicates the other leg of the x-type current structure. However, the plotted cross-section is not perpendicular to this part and hence, we only concentrate on the section between the dotted lines.

One of the basic characteristics of a slow mode shock is that the magnetic field strength is refracted towards the shock normal This implies that the size of the tangential component of the field decreases as the shock front passes, and Fig. 11 shows that this property is indeed satisfied (the tangential component is given by $B_{y}$ - thick dashed line). The normal magnetic field component ( $B_{x}$ - thick dot-dashed line) remains more or less constant across the current structure. Looking at the vorticity, there is a clear peak, indicating a rapid change in the velocity at this particular location. However, examining the individual velocity components, we find that these do not conform with the standard definition of a slow shock. A slow shock is expected to slow down the tangential component of the flow but from Fig. 11 (thin dashed line), we see that in this case the tangential component actually increases, as the outflow velocities from the reconnection region are directed along the current structure. Additionally, the observed velocities on both sides of the shock are significantly slower than the local Alfvén and sound speeds. Overall, although both the current structure and the magnetic field components resemble a slow shock, it appears that the velocities are too small to actually form a shock.

Finally, we investigate the nature of the bowshaped currents. The cross-sections in the right-hand graph of Fig. 11 are taken at mid-height, $z=0.5$ and along constant $x=0.5$. This again ensures that the part of the cross-sections between the vertical dotted lines is normal to the current structure at those particular locations. The tangential component of the magnetic field ( $B_{x}$ - thick dot-dashed line) is again reduced across the structure, pointing towards a slow shock. However, the normal component of the magnetic field ( $B_{y}$ - thick dashed line) is almost zero, with only a very small change. If the normal component of the magnetic field is identically zero, a slow shock reduces to a tangential discontinuity. The constant total pressure is also consistent with a tangential discontinuity. Looking at the normal component of the velocity ( $v_{y}$ - thick dashed line), it is hard to decide whether the change in $v_{y}$ constitutes a jump, or is just part of the decreasing velocity across the reconnection outflow region. Again the magnitude of the velocity is much lower than the Alfvén and sound speed.

\section{Connectivity and reconnection rates}

The connectivity changes, and the amount of flux associated with them, are calculated following the method used by Parnell \& Galsgaard (2004). A large number of fieldlines (5968) are traced from each of the flux concentrations at the lower boundary. During the rotational driving, the shape of the sources remains largely unchanged, with the fieldlines frozen into the plasma and hence, each of the traced fieldlines can be associated with a fixed amount of flux. Therefore, as fieldlines change connectivity, the amount of "reconnected" flux can be calculated. Snapshots of the structure of the fieldline connectivity for one of the sources, taken at various stages during both the potential and the dynamical evolution, are shown in Fig. 12. Fieldlines have been traced from within a radius $r_{0}$ of the centre of the source on the bottom boundary. All fieldlines that end up within a radius $2 r_{0}$ from the centre of a source on the top boundary are assumed to be connect to that particular source. Fieldlines still at their original connection are coloured pale blue, whereas fieldlines that have reconnected to the other flux concentration are represented in dark blue. White corresponds to fieldlines which are connected with parts of the top boundary not associated with the flux concentrations (i.e. they end up outside the $2 r_{0}$ radius, defined above) and red fieldlines are leaving through the sides of the box. The direction of the centre of rotation is indicated by a solid black line in the snapshots on the top row. As the snapshots in the middle and bottom row are taken at approximately the same values of $\theta$, the black lines have been omitted to not needlessly overcrowed the graphs. 

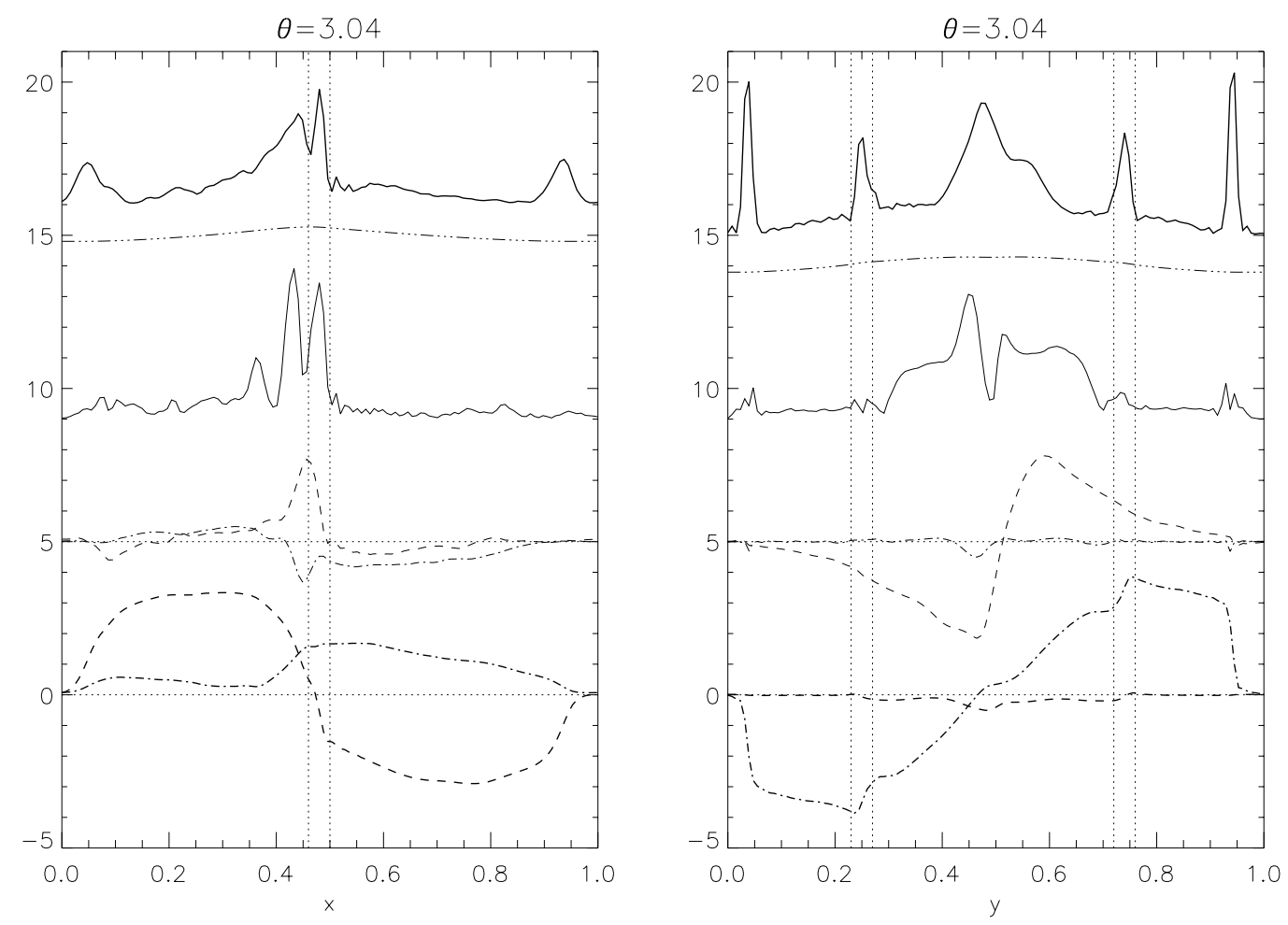

Fig. 11. (left) Cross-section at $y=0.6$ and $z=0.4$ of current (thick solid), vorticity (thin solid), total pressure (dot-dot-dashed), $v_{x}$ (thin dot-dashed) and $v_{y}$ (thin dashed) and $B_{x}$ (thick dot-dashed) and $B_{y}$ (thick dashed) for $B_{\mathrm{bg}}=0.05$. Units on the $y$-axis are arbitrary. The horizontal dotted lines outline the appropriate zero levels for the velocity and magnetic field components. The vertical dotted lines outline the position of one of the legs of the x-type structure. (right) Cross-section at $x=0.5$ and $z=0.5$, with the vertical dotted lines outlining the position of the bowshaped structures.

For comparison, the corresponding "potential” connectivity evolution is also shown in Fig. 12 (top row). At each time step, the potential field was calculated and the fieldline connectivity of the sources was determined as above. The evolution of the potential connectivity is relatively easy to understand. As the potential field corresponds to the lowest energy stage, the original connectivity structure (pale blue) is regained every time the initially aligned sources are realigned, i.e. at every rotation (and counter-rotation!) angle that is a multiple of $\pi$. Whenever the rotational angle is an odd multiple of $\pi / 2$, the connectivity of the flux sources has entirely reversed (dark blue). At intermediate stages, part of the fieldlines connect to either of the sources and a small number connects to a neighbouring (periodic) domain (red). The white fieldlines do connect to the flux concentrations on the top boundary but fall just outside the $2 r_{0}$ radius. When a background field is present, a large amount of the potential fieldlines connects to parts of the top boundary not associated with the flux concentrations whenever those are not almost exactly aligned. This is again due to the fact that the fieldlines look for those connections that represent the lowest energy state.

The next 2 sequences of snapshots (middle \& bottom row) show that the dynamical evolution of the structure of the connectivity is far from trivial. The portion of the flux concentration that has reconnected, i.e. the dark blue segment, is mainly on the "leading" edge of the source. These fieldlines are situated closest to the twisted current sheet and hence, the (quasi-)separatrix surface. The fraction of reconnected flux becomes increasingly large as the sources are rotated further, extending simultaneously along the edge of the source and towards its centre. Although this study models a different type of boundary driving, the evolution of the footpoint connectivity shows many similarities with the experiments described by Parnell \& Galsgaard (2004). When driving two, initially unconnected, flux sources past each other, they also found that the flux on the leading edges closes (reconnects) first. In the later stages of the evolution, a substantial amount of fieldlines (coloured red) are connecting to the neighbouring (periodic) domain, due to the absence of a confining background field and hence, they are seen as leaving through the sides of the numerical box. Rather than growing inwards towards the centre of the source, this red segment remains mainly near the edge of the flux sources as those fieldlines are closest to the boundaries of the domain and hence, to the neighbouring (periodic) flux concentrations. Note that in this case $\left(B_{\mathrm{bg}}=0\right)$, relatively few white fieldlines are present. The small number that can be seen in the middle row of Fig. 12) again correspond to fieldlines which connect to the sources on the top boundary, but fall just outside the $2 r_{0}$ radius.

When a background field is present, the evolution of the connectivity is equally complex and shows many similarities with the $B_{\mathrm{bg}}=0$ case. However, at most stages, far less (red) fieldlines are seen to be leaving through the sides of the domain, due to the confining effect of the background magnetic field. Although the entire top boundary is now permeated by a magnetic field, most of the fieldlines do end up connecting to a flux source again, unlike the corresponding potential evolution, where a large fraction of the flux connects to parts of the top boundary not associated with the flux concentrations. However, at the end of the experiment, a very large fraction of fieldlines traced from the flux concentrations reconnect to the neighbouring domain (final figure, bottom row Fig. 12), due to the large current concentrations on the side of the box, which form when the outward propagating "bow" currents collide with the ring of current at the edge of 

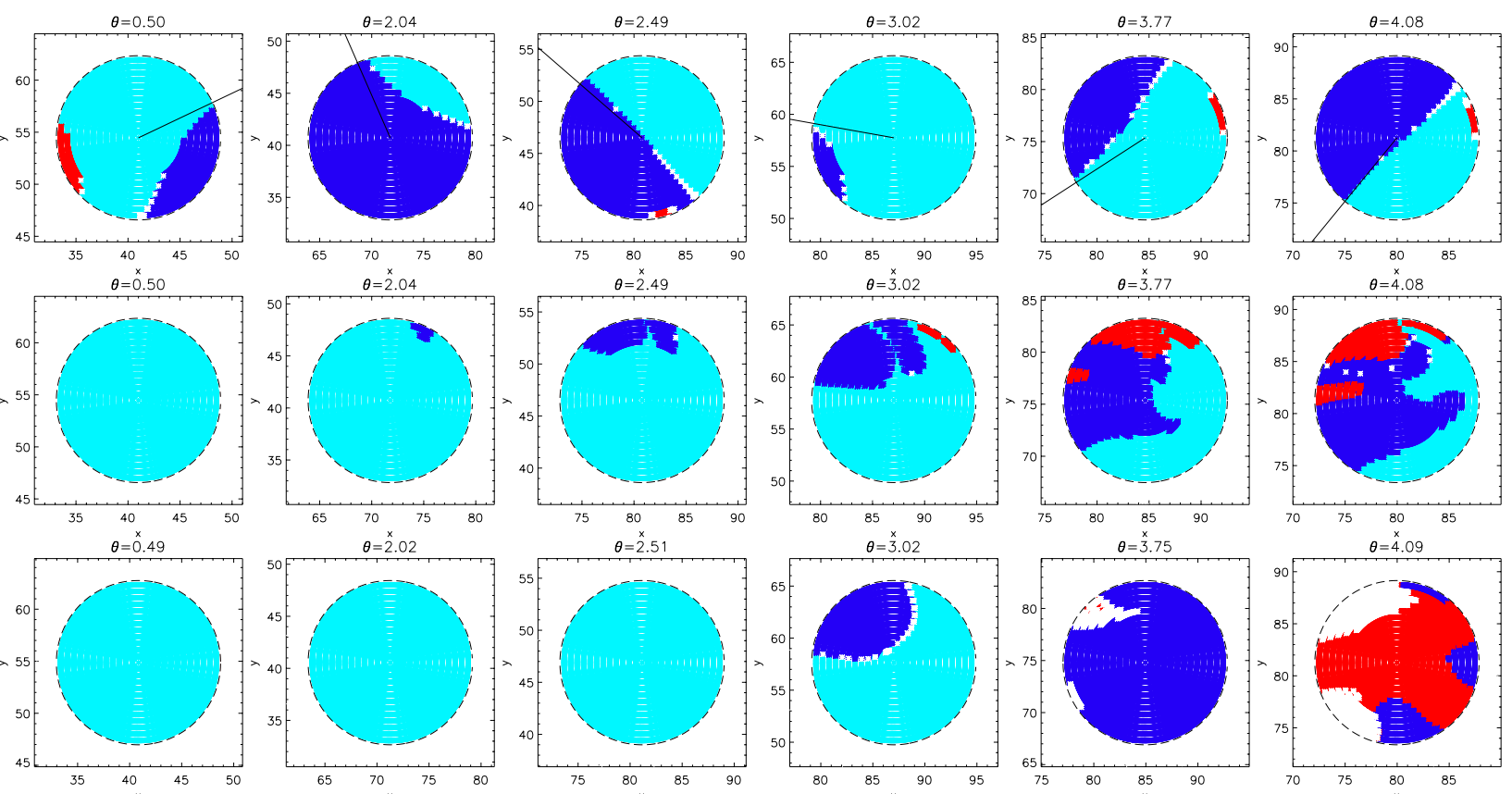

Fig. 12. The fieldline connectivity of one of the sources on the lower boundary, at different rotational angles, for the potential evolution with $B_{\mathrm{bg}}=0$ (top row) and the dynamical evolution with $B_{\mathrm{bg}}=0$ (middle row) and $B_{\mathrm{bg}}=0.05$ (bottom row). The pale blue colour corresponds to fieldlines at their original connection, the dark blue shows fieldlines that have reconnected to the other flux source whereas the red corresponds to fieldlines that are leaving through the sides of the box and white are fieldlines connected with parts of the top boundary not associated with the flux concentrations. The solid black lines in the top row indicate the direction of the centre of rotation.

the domain. At this stage, a significant number of fieldlines also connects to parts of the top boundary not associated with the flux concentrations.

The amount of reconnected flux from one of the sources, as a percentage of the total flux of that source, is plotted in Fig. 13, both for fieldlines changing their connection to the other flux source (left - dark blue in Fig. 12) and (right) fieldlines that are connecting to parts of the top boundary not associated with the flux concentrations (thick lines - white in Fig. 12) or to neighbouring (periodic) domains (thin lines - red in Fig. 12). The potential and dynamical evolution are shown in the top and bottom row, respectively. The solid lines correspond to the system without a background field, whereas the dashed and dotdashed lines correspond to $B_{\mathrm{bg}}=0.025$ and $B_{\mathrm{bg}}=0.05$, respectively. The cyclical pattern of the potential connectivity described above is clearly evident from Fig. 13 (top). At every odd multiple of $\pi / 2$, the flux reconnected to the other flux source reaches $100 \%$ (i.e. all the flux from one source has now reconnected to the other source). On the other hand, at every multiple of $\pi$, both the amount of flux reconnected to the other source, as well as the flux connected to other parts of the top boundary or to a neighbouring domain, reaches zero (i.e. all the flux has returned to the original connection). From Fig. 13 (top right), it can be seen that for the potential $B_{\mathrm{bg}} \neq 0$ case, no fieldlines leave through the side of the numerical domain, unlike in the $B_{\text {bg }}=0$ case. Note also that the amount of reconnected potential flux actually reaches $100 \%$, which is never attained in the dynamical experiments. Finally, we point out that the increase in reconnected flux does not start as soon as the boundary driving is switched on, suggesting that it is possible to rotate the sources by a very small amount without altering the potential field configuration.

As expected, the dynamical evolution is very different, with the substantial delay of the onset of reconnection being the most striking feature. Comparing to the $B_{\mathrm{bg}}=0$ case, reconnection between the fluxtubes starts later when a stronger background field is present, i.e. at a larger rotational angle. As the fluxtubes are more confined, it takes time before the initially isolated fluxtubes come into contact. However, as was already evident from Table 1, reconnection takes place faster as the strength of the background field is increased. This apparent difference in reconnection rates between the various cases can be attributed to the increasing confinement of the fluxtubes, which leads to a higher flux density when the fluxtubes finally enter into the current sheet. This results in a higher current magnitude and subsequently, an increase in the reconnection rate. Comparing the results for $B_{\mathrm{bg}}=0$ and $B_{\mathrm{bg}}=0.05$, we also notice that a larger fraction flux connects to the other source - just over $70 \%$ at the time where the $B_{\mathrm{bg}}=0$ experiment is stopped (before reaching its absolute peak) compared to a peak of $95 \%$ for $B_{\mathrm{bg}}=0.05$.

Finally, Fig. 13 (right - thin solid lines) shows clearly that a larger amount of flux now connects to neighbouring domains (fieldlines leaving through the periodic boundaries) when the confining background field is absent. However, this situation changes dramatically in the final stage of the experiments. Unlike the potential $B_{\mathrm{bg}} \neq 0$ case, a very large proportion of the $B_{\mathrm{bg}} \neq 0$ fieldlines (thin dot-dashed \& dashed lines) now connect to the neighbouring periodic domain. Note that for $B_{\mathrm{bg}}=0$ and $B_{\mathrm{bg}}=0.025$, the percentages of flux reconnected to the other source and flux reconnected elsewhere do not at up to $100 \%$ at any give time, as a small fraction of the flux remains at its original connection. Only in the $B_{\mathrm{bg}}=0.05$ case does $100 \%$ of the flux associated with the sources on the lower boundary change connection. (see also Table 1).

\section{Energetics}

In the experiments, the magnetic configuration is stressed by imposing a particular boundary driving profile, Eq. (2), with a given amplitude. This boundary driving imposes a work on the 

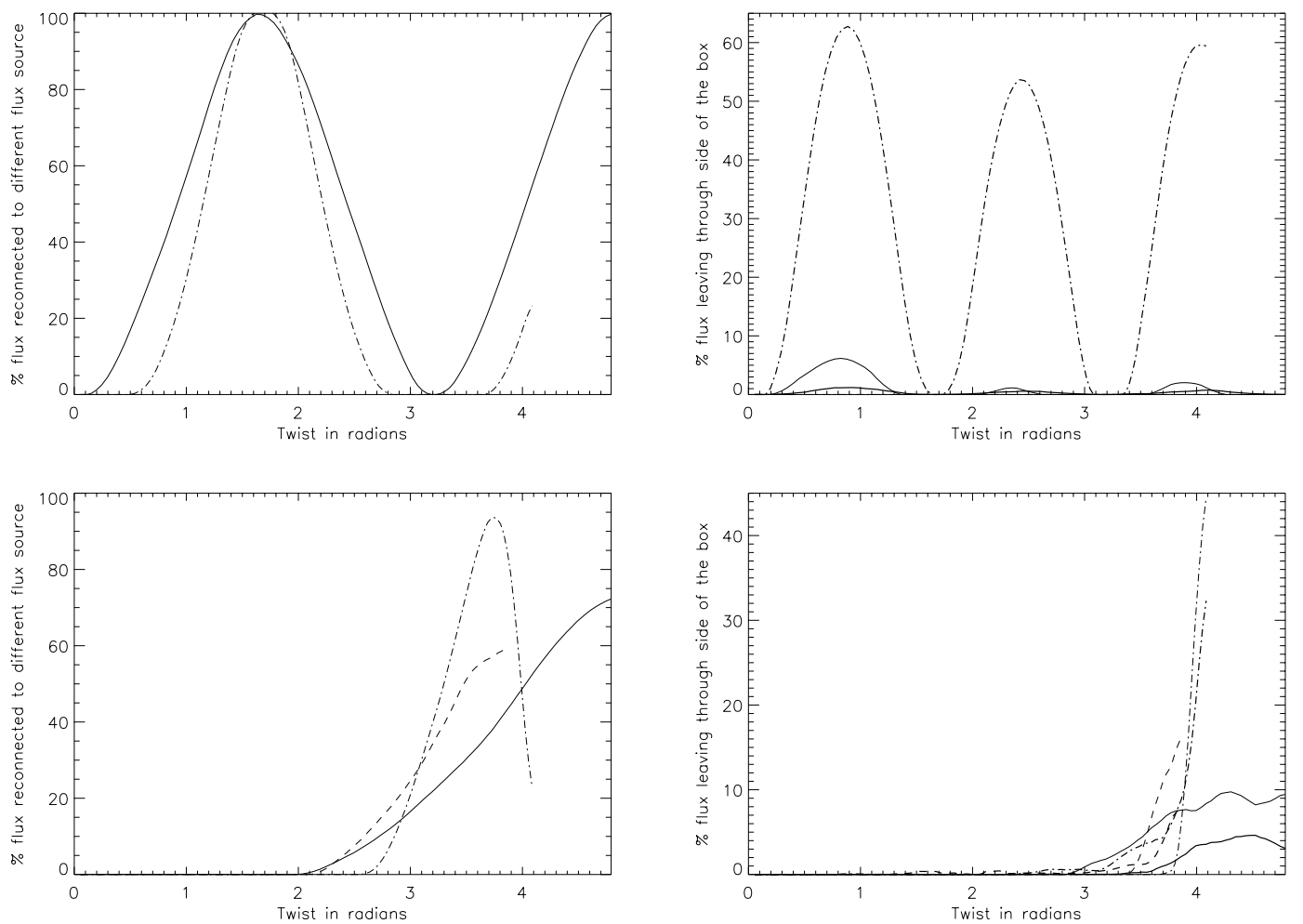

Fig. 13. The evolution of the flux, as a percentage of the total flux in the source, (left) reconnected to a different source and (right) the flux associated with fieldlines leaving the sides of numerical box (thin lines) or connecting to parts of the top boundary not associated with the flux concentrations (thick lines) both for the potential (top row) and dynamical (bottom row) evolution. Solid, dashed and dot-dashed lines represent $B_{\mathrm{bg}}=0, B_{\mathrm{bg}}=0.025$ and $B_{\mathrm{bg}}=0.05$, respectively.
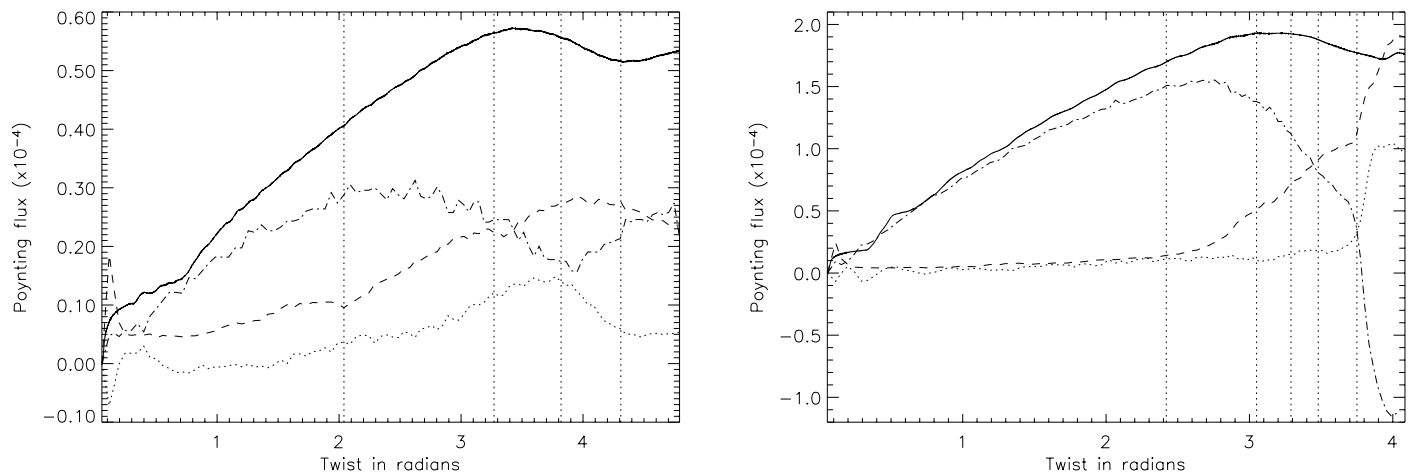

Fig. 14. The Poynting flux as a function of rotation angle, for the case $B_{\mathrm{bg}}=0($ left $)$ and $B_{\mathrm{bg}}=0.05($ right $)$. The dot-dashed line shows the change in magnetic energy, the dashed line is the Joule dissipation and the dotted line represents the work done by the Lorentz force. The vertical dotted lines represent the angles at which reconnection starts, $25 \%, 50 \%, 75 \%$ and $100 \%$, respectively, of the flux have reconnected in each case.

magnetic field, but it does not determine how large this work is or how it changes in time. This is determined by the rearrangement of the magnetic field inside the domain and the relative angles of the magnetic field at the driving boundaries. To understand the dynamical evolution of the system it is of interest to investigate the energy flow in the system.

The Poynting flux (Fig. 14) behaves very similarly in the case with and without a vertical background field. The magnitude of the Poynting flux is about 3 times larger for the $B_{\text {bg }}=0.05$ case. This is due to the much larger magnetic area that is stressed when the entire boundary is covered with a magnetic field. The Poynting flux represents the magnetic energy that passes through the driving boundaries. To know which form this energy takes, we rewrite the Poynting flux as three volume contributions to the change in magnetic energy density in the $3 \mathrm{D}$ domain: the rate of change of the magnetic energy (dot-dashed line), the ohmic heating (dashed line) and the work done by the Lorentz force (dotted line).

Qualitatively, the evolution of the overall energetics of the experiments can be summarised as follows. As the rotational driving of the sources starts, the magnetic field becomes twisted, resulting in an increase of the magnetic energy. Additionally, current concentrations start to form and hence the joule dissipation builds up slowly. This process continues until the onset of reconnection (first dotted, vertical line), which releases some of the tension stored in the magnetic field and hence, the increase 

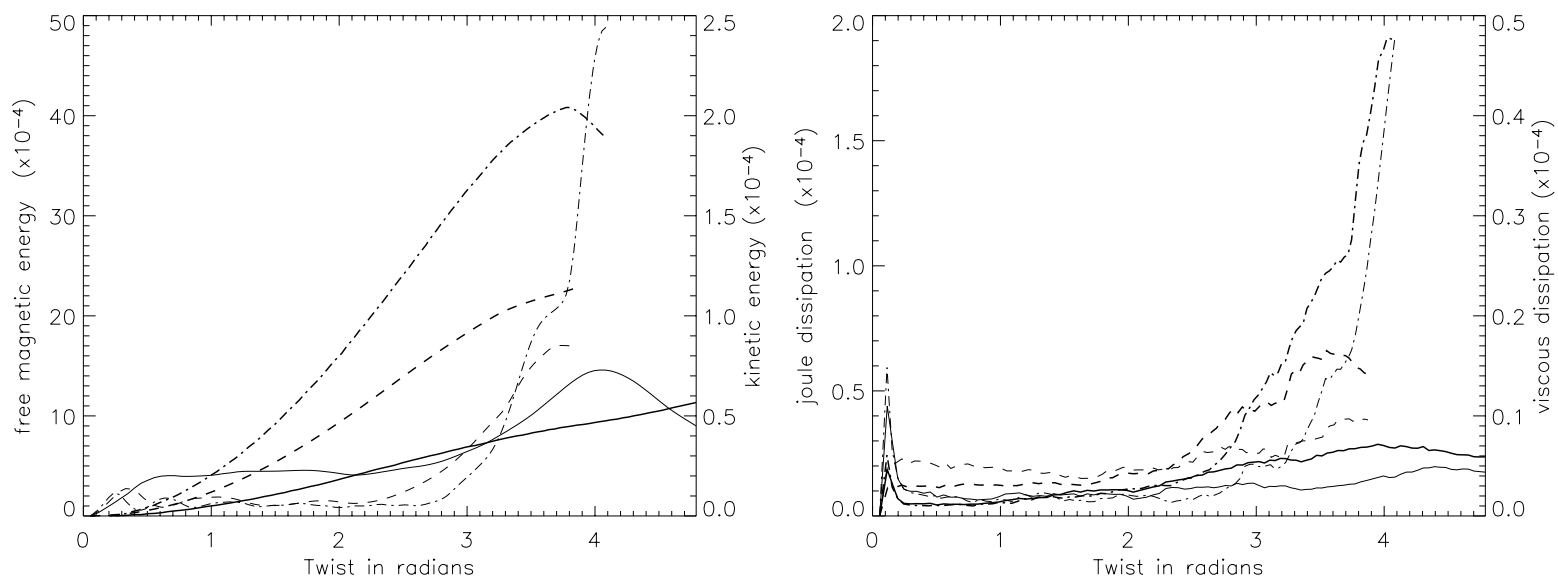

Fig. 15. (left) The free magnetic energy (thick lines) and the kinetic energy (thin lines) as a function of rotation angle, for the case $B_{\mathrm{bg}}=0$ (solid line), $B_{\mathrm{bg}}=0.025$ (dashed line) and $B_{\mathrm{bg}}=0.05$ (dot-dashed line). (right) The Joule (thick lines) and viscous (thin lines) dissipation.

of magnetic energy is slowed down. The strong current concentrations that are now present result in a more rapid increase in the ohmic heating term. As plasma is ejected from the reconnection region, the kinetic energy (see Fig. 15) and hence, the viscous dissipation, start to build up. This is reflected in the increase in the work done by the Lorentz force.

The next change in behaviour takes place after about $50 \%$ of the flux has reconnected (third dotted vertical line) and at this stage, there are marked differences between the case without and with background field. When no background field is present, reconnection appears to slow down, resulting in a decline of the work done by the Lorentz force, the kinetic energy and both the ohmic and viscous dissipation. However, as the rotational driving is still ongoing, the reconnected field is now becoming increasingly stressed again, resulting in a renewed increase in the magnetic energy and effectively, the whole process would start over again, if the experiment was continued further. When a vertical magnetic field is added, the situation is different, as reconnection appears to slow down only slightly. In the final stage, when all the flux has reconnected (last dotted vertical line), the current concentrations at the head of the oppositely directed reconnection outflows (see Fig. 8 - bottom) collide with the ring of current near the outside of the domain, thus forming large current concentrations on the edges of the numerical domain. Due to the periodic boundary conditions, these cause the magnetic field to reconnect again and at the end of the experiment, most of the magnetic fieldlines traced from the flux concentrations on the lower boundary leave through the sides of the box. The effect of this dramatic opening of the fieldlines is evident from the very rapid increase in both the ohmic heating and the work done by the Lorentz force. At the same time, most of the magnetic tension is released, resulting in a turnover of the magnetic energy.

Figure 15 (left) shows the free magnetic energy (thick lines) and the kinetic energy (thin lines) for three different values of the background field. The free magnetic energy is determined by subtracting the magnetic energy of the corresponding potential state. It is obvious from this graph that both the free magnetic energy and the kinetic energy reach larger values when the strength of the background magnetic field is increased: both energies increase more than three fold for the $B_{\mathrm{bg}}=0.05$ case, compared to the no-background case. Comparing the (free) magnetic and kinetic energy shows that the former is more than an order of magnitude larger, regardless of the magnitude of the background magnetic field. Analogous results are found in both dissipative processes (Fig. 15 - right), with the Joule dissipation (thick lines) almost an order of magnitude larger than the viscous dissipation (thin lines). Note that a weaker background magnetic field, $B_{\mathrm{bg}}=0.025$, only results in a surprisingly small increase in the kinetic energy and the viscous dissipation.

\section{Discussion}

From a topological point of view, the two sets of experiments $\left(B_{\mathrm{bg}}=0\right.$ and $B_{\mathrm{bg}} \neq 0$ ) investigated in this study are substantially different. Indeed, when a background magnetic field is absent, independent regions of magnetic connectivity, divided by separatrix surfaces, are present in the domain. However, when a constant, background magnetic field is added, there are no distinct connectivity regions, as the whole boundary contains flux of the same polarity, and initially, only a weak quasi-separatrix layer is present. Despite this obvious topological difference, the overall, dynamical evolution of both models showed many similarities: frozen into the plasma, the initially straight magnetic field is deformed into a helical-like structure due to the rotational motion of the flux concentrations on the boundaries. The increasing magnetic tension results in a Lorentz force, compressing the central region of the domain. These tensions in the magnetic field initiate a stagnation point flow, gradually building up a current sheet along the (quasi-)separatrix surface. This helical magnetic field structure, wrapped around a central (quasi-)separator current sheet, was also found by Priest et al. (2005), who investigated essentially the same configuration $\left(B_{\mathrm{bg}}=0\right)$ analytically, using a reduced MHD model. Eventually, the currents at the (quasi-)separatrix layer are sufficiently large to allow for the onset of reconnection, changing the footpoint connectivities. A comparison with the corresponding potential evolution showed a large delay in the onset of reconnection, as the dynamical evolution requires the build up of sufficiently strong currents before reconnection can take place. Regardless of the value of the background magnetic field, the outflow velocities from the reconnection region are found to be relatively weak, and at all times, remain smaller than the local Alfvén and sound speeds.

Although the global, dynamical evolution is similar, there are some important differences between the background and nobackground cases, mainly in the timing and the amount of energy that is released. Firstly, when a background field is present, 
the activity observed in our simulations is much more energetic. This is not unexpected, as the rotational boundary driving now stresses a much larger surface area containing a normal component of the magnetic field, resulting in a higher Poynting flux through the boundaries of the domain and hence, more free magnetic energy will be available for release. Additionally, as the background magnetic field was simply added to the existing sources, the $B_{\mathrm{bg}} \neq 0$ case contains a higher amount of magnetic flux, even before the boundary driving has started. Secondly, the presence of a background field delays the onset of reconnection between the fluxtubes, as reconnection first has to remove the background flux which keeps the two fluxtubes apart. Although it takes longer for the fluxtubes to come into contact, the increased confinement leads to a higher flux density, which in turn results into a higher current and hence, faster reconnection. The fact that there are no distinct connectivity regions present in the domain at the start of the experiment seems to have little influence on the overall evolution. When $B_{\mathrm{bg}} \neq 0$, a QSL is present in the central region before the boundary driving starts, but it is relatively weak and broad. As the sources are rotated, a stagnation flow is established, carrying the two fluxtubes toward the central region. This will result in a steepening of the connectivity change at the central region and hence, the QSL becomes increasingly narrow and steep. As the strength of the background magnetic field is increased, the initial QSL is broader, with smaller values of the squashing factor $Q$. However, at the same time, the currents that eventually build up at the QSL, reach higher values for larger values of $B_{\mathrm{bg}}$. This results agrees with the findings of Aulanier et al. (2005), that currents reach higher magnitudes in configurations which initially contain broader QSLs.

In the solar atmosphere, the fact that the more energetic activity is occurring at QSLs, compared to (real) separatrix surfaces, might have significant implications. Indeed, as virtually the entire solar atmosphere is threaded by magnetic field, it is likely that the more energetic QSLs outnumber real separatrix surfaces, which require the presence of magnetic nulls (regions where the magnetic field vanishes). In this respect, the experiments that contain a non-zero magnetic field are probably the more realistic ones, with the background magnetic field, and the field associated with stronger flux concentrations subjected to (photospheric) footpoint motions. However, the experiments studied here have some significant shortcomings, making a direct comparison with realistic, solar, situations somewhat difficult. Most importantly, the model atmosphere contains an initially constant pressure, resulting in plasma $\beta$ values that are much higher than in the solar corona. Away from the flux concentrations on the boundaries, $\beta>1$, whereas in reality, the coronal $\beta$ is expected to be smaller than one. This causes the Alfvén speed to be smaller than the sound speed, and the gas pressure to dominate the magnetic pressure in the central parts of the domain, i.e. where the reconnection is occurring. This shortcoming could be addressed by including gravitational stratification, which would give a more realistic distribution of $\beta$ values throughout the domain, i.e. maintain $\beta>1$ near the footpoints but $\beta<1$ at the reconnection sites. This is likely to increase the reconnection outflow velocities but we do not expect any significant changes to the overall dynamical evolution presented in the current study.

The periodic boundary conditions at the sides of the numerical box can be considered to be an additional shortcoming. As it allows the fieldlines to connect to the neighbouring periodic domain, it significantly complicates the dynamical evolution, especially in the later stages of the experiments. When no confining background magnetic field is present, some flux reconnects through the boundaries throughout the experiment. However, at the same time, this periodic setup can be seen as more realistic than an isolated numerical domain, as the photospheric magnetic carpet is full of flux concentrations. In the context of the coronal tectonics model of Priest et al. (2002), coronal loops connect to the photosphere in a multitude of different magnetic fragments. The two fluxtubes in our experiments can be seen as two individual loops strands, with the loop strands in the neighbouring domains making up the rest of the coronal loop. Hence, it is more naturally to allow not only for interactions between the fluxtubes inside the numerical domain, but also with the surrounding loops strands through the periodic boundary conditions.

Complex motions of solar magnetic fragments can be decomposed into a translation and shear of the sources, a spinning of each of the sources and a rotation of the sources about each other. A simple translation does not result in the build up of current sheets, whereas shearing, spinning and rotational motions of a pair of sources will generate separatrix and separator current sheets, respectively (Titov et al. 2003; Priest et al. 2005). The footpoint motions considered in this study were limited to a purely rotational motion of the flux sources. Obviously, such footpoint motions are not representative of real solar motions but were designed to isolate (quasi-)separator reconnection. Reconnection between the fluxtubes started at a rotational angle of $\theta=2.04-2.42$, depending on the value of the background magnetic field. As the top and bottom boundaries were counter-rotated, the fluxtubes have been twisted by $1.3-1.5 \pi$. At the end of the experiments, the sources on both top and bottom boundaries were rotated as much as $\theta=4.78$, resulting in a very large total twist of almost $3 \pi$. Indeed, studying the behaviour of a single coronal X-ray bright point, Brown et al. (2001) estimated that the relative motions of the magnetic fragments contain a rotation with an angle of only about $\pi / 5$. Hence, the angles considered in our experiments are likely to be much larger than realistic, solar values. On the other hand, the initial field configuration in our experiments is potential and hence, it is not surprising that a large amount of rotation is needed to build up sufficiently large stresses in the field.

Despite the various shortcomings described above, it is a worthwhile exercise to put the results of our study into a solar context, by using the relations (9)-(14). Let us choose the unit of magnetic field strength $B_{0}=6 \mathrm{G}$, the electron number density $n_{\mathrm{e}}=10^{15} \mathrm{~m}^{-3}$ and the unit of length $l_{0}=77 \mathrm{Mm}$. This choice results in a velocity $v_{0}=414 \mathrm{~km} \mathrm{~s}^{-1}$, time $t_{0}=186 \mathrm{~s}$ and temperature $T_{0}=1.2 \times 10^{7} \mathrm{~K}$. Given this set of unit quantities, let us first look at the initial setup of the models, i.e. the value of the various parameters at $t=0$. For notational convenience, barred variables represent the (dimensionless) values used in the numerical code. At the start of the numerical experiment, we have

$\bar{\rho}_{(t=0)}=0.1 \Rightarrow \rho_{(t=0)} \approx 1.7 \times 10^{-13} \mathrm{~kg} / \mathrm{m}^{3}$,

$\bar{T}_{(t=0)}=\gamma=5 / 3 \Rightarrow T_{(t=0)} \approx 8.3 \times 10^{5} \mathrm{~K}$,

$\bar{p}_{(t=0)}=0.1 \gamma \Rightarrow p_{(t=0)} \approx 2 \times 10^{-3} \mathrm{~Pa}$,

$\bar{B}_{\max (t=0)}=0.89 \quad \Rightarrow \quad B_{\max (t=0)} \approx 5.4 \mathrm{G}$,

$\bar{B}_{\mathrm{bg}(t=0)}=0.05 \quad \Rightarrow \quad B_{\mathrm{bg}(t=0)} \approx 0.3 \mathrm{G}$,

$\bar{v}_{\text {driver }}=0.02222 \Rightarrow v_{\text {driver }} \approx 9 \mathrm{~km} \mathrm{~s}^{-1}$,

$\bar{r}_{0}=0.065 \Rightarrow r_{0} \approx 5 \mathrm{Mm}$,

where $B_{\max }$ is the maximum magnetic field strength of the sources and $r_{0}$ is the radius of the sources. Although the experiments were not designed with any particular solar structure in mind, the size and magnetic field strength of the sources could be seen as representative of X-ray bright Points (XBP's). 
Table 2. An overview of the time (in hours) at which various stages of the reconnection occur, for different values of the additional background magnetic field, $B_{\mathrm{bg}}$.

\begin{tabular}{lccc}
\hline \hline & $B_{\mathrm{bg}}=0$ & $B_{\mathrm{bg}}=0.025$ & $B_{\mathrm{bg}}=0.05$ \\
\hline Onset of reconnection & 1.15 & 1.21 & 1.37 \\
25\% reconnected flux & 1.87 & 1.70 & 1.74 \\
$50 \%$ reconnected flux & 2.18 & 1.96 & 1.87 \\
$75 \%$ reconnected flux & 2.47 & 2.13 & 1.99 \\
$100 \%$ reconnected flux & - & - & 2.14 \\
End of experiment & 2.75 & 2.16 & 2.32 \\
\hline
\end{tabular}

The temperature, density and pressure correspond to typical (low) coronal values. To get a feel for the timescales, Table 2 again gives the various stages of the reconnection process but now in terms of real times (in hours). For example, when no background field is present, we see that the onset of reconnection occurs after $1.15 \mathrm{~h}$. Or, when a background field of $0.3 \mathrm{G}$ is present $\left(B_{\mathrm{bg}}=0.05\right)$, all the flux has reconnected after $2.14 \mathrm{~h}$. Comparing Tables 1 and 2 now allows the determination of all the relevant timescales.

Finally, we look into the energy that would be released by the reconnection events described in this study. The free magnetic energy (integrated over volume), shown in Fig. 15 (left), reaches maximum values of the order of $(1.1-4.1) \times 10^{-3}$, depending on the value of the background magnetic field. Or, using the values for $B_{0}, \mu_{0}$ and $l_{0}$ described above, the free magnetic energy (integrated over the volume) reaches a maximum of about (1.5-5.3) $\times 10^{27} \mathrm{erg}$, approximately $2.1-2.3 \mathrm{~h}$ after the onset of the rotational driving $(\theta=3.8-4)$. Although the free magnetic energy is the energy in the system that is available for release, it is clear from comparing Fig. 15 (left) and (right), that not all this energy is actually released during the experiments. Hence, a more relevant quantity to look at is the amount of dissipated energy, i.e. the total Joule and viscous dissipation, integrated over time. Again depending on the strength of the background magnetic field, the total dissipated energy reaches values of $(1.3-2.5) \times 10^{27} \mathrm{erg}$ by the end of the respective experiments. This is of the order of various small-scale heating events, such as XBP's or Active Region Transient Brightenings (ARTB's). A discussion of the relevance of such small-scale heating events, with respect to the overall coronal heating, is beyond the scope of this paper and for a comprehensive discussion, we refer the interested reader to Parnell (2002) and references therein. Note that the amount of dissipated energy will depend on the form of resistivity and viscosity used in the numerical experiments, as was pointed out by Mellor et al. (2005). Choosing a different set of unit values for $B_{0}, n_{\mathrm{e}}$ and $l_{0}$ will of course also result in a different range of dissipated energies.

Future investigations will focus on a variety of aspects. An interesting parameter which was not varied in the experiments presented in this study, is the amplitude of the rotational driver. Galsgaard \& Parnell (2005) found that the most important factor is the distance the flux sources are advected, not the speed at which they are driven. When considering a rotational driver, it would be useful to compare the rotational angle of the onset of reconnection when the driving speed is varied. Additionally, the initially aligned flux concentrations on the top and bottom boundary result in a very symmetrical configuration which is unlikely to be realistic. This will be addressed by considering a different placement of the flux concentrations, e.g. at a 90 degree angle, as in Titov et al. (2003) and Galsgaard et al. (2003).
Finally, a comparison will be made between different types of boundary driving of the flux sources. Priest et al. (2005) considered both rotation and spinning of two adjacent fluxtubes, in order to compare the build up of current sheets and hence, coronal heating, at both separators and separatrix surfaces. So far, we have only considered the rotational driving of the flux sources, leading to separator reconnection. In a next set of experiments, the rotational driver will be modified to study the spinning of the flux concentrations, which will allow us to compare the build up of current, as well as the rate of reconnection, at separators and separatrix surfaces.

\section{Conclusions}

In this paper, we presented results of 3D numerical simulations of magnetic reconnection driven by simple, rotational footpoint motions, concentrating mainly on a qualitative description of the overall evolution of the experiments. The opposite footpoints of two, initially straight fluxtubes are counter-rotated, resulting in the rapid build up of a central, twisted current sheet at the (quasi-)separatrix surface. As reconnection takes place, the footpoint connectivity changes in a non-trivial manner, showing very little resemblance to the cyclical nature of the corresponding potential experiments, which dramatically oversimplify the evolution of the interacting fluxtubes. Overall, the evolution of the systems with and without a constant, background magnetic field is very similar, with the QSLs in the $B_{\mathrm{bg}} \neq 0$ case fulfilling much the same role as the separatrix layers in the $B_{\mathrm{bg}}=0$ case.

Although these QSLs are initially present in the $B_{\mathrm{bg}} \neq 0$ cases, the stresses imposed on the magnetic field by the boundary rotation cause a substantial increase in the strength of the QSL (i.e. in the value of the squashing factor $Q$ ), as well as reducing their width. A stronger background magnetic field increases the confinement of the fluxtubes and hence, is associated with a broader and weaker initial QSL. Although this increased confinement of the fluxtubes causes a delay in the onset of reconnection, the rate of reconnection is higher for larger values of the background field, due to the higher flux density in the fluxtubes. Additionally, the amount of free magnetic energy available for release is higher when a background magnetic field is present. As the solar atmosphere is permeated by magnetic fields, the more energetic activity at QSLs is likely to be the more realistic scenario. Although the experiments were not designed to model any particular solar structure, assuming reasonable initial coronal values resulted in an energy release of about $10^{27} \mathrm{erg}$, which is of the order of a variety of small-scale heating events such as XBP's or ARTB's.

Although there are certain similarities, these 3D numerical simulations show that, not unexpectedly, there are substantial differences between reconnection in $3 \mathrm{D}$ and the simple 2D model. Looking at the midplane of the numerical domain, the current showed the "traditional" $x$-type structure, but the helical magnetic field actually forms an O-point configuration, despite the presence of a stagnation point flow. Also, only remnants of the slow shocks often associated with $2 \mathrm{D}$ reconnection were found: the current configuration looks like a slow shock structure and the magnetic field contains the signature of a slow shock but the associated velocities are too small to actually form a shock.

Acknowledgements. I.D.M. acknowledges support of a Royal Society University Research Fellowship. K.G. was supported by the Carlsberg Foundation in the form of a fellowship. The authors would like to thank Profs. A. W. Hood and E. R. Priest for helpful discussions and the referee for useful suggestions. 


\section{References}

Aulanier, G., Pariat, E., \& Démoulin, P. 2005, A\&A, 444, 961

Brown, D. S., Parnell, C. E., DeLuca, E. E., Golub, L., \& McMullen, R. A. 2001, Sol. Phys., 201, 305

Close, R. M., Parnell, C. E., \& Priest, E. R. 2004a, Sol. Phys., 225, 21

Close, R. M., Parnell, C. E., Longcope, D. W., \& Priest, E. R. 2004b, ApJ, 612, L81

Démoulin, P., Hénoux, J. C., Priest, E. R., \& Mandrini, C. H. 1996, A\&A, 308, 643

Démoulin, P. 2005, Adv. Space Res., in press

Falconer, D. A., Moore, R. L., Porter, J. G., \& Hathaway, D. H. 2003, ApJ, 593, 549

Galsgaard, K., \& Nordlund, A. 1996, JGR, 101, 13445

Galsgaard, K., \& Nordlund, ̊.. 1997, JGR, 102, 231

Galsgaard, K., Parnell, C. E., \& Blaizot, J. 2000, A\&A, 362, 395

Galsgaard, K., \& Parnell, C. E. 2005, A\&A, 439, 335

Galsgaard, K., Priest, E. R., \& Nordlund, Å. 2000, Sol. Phys., 193, 1

Galsgaard, K., Titov, V. S., \& Neukirch, T. 2003, ApJ, 595, 506

Gudiksen, B. V., \& Nordlund, Å. 2005, ApJ, 618, 1031

Hagenaar, H. J. 2001, ApJ, 555, 448

Hagenaar, H. J., Schrijver, C. J., \& Title, A. M. 2003, ApJ, 584, 1107

Haynes, A., Parnell, C. E., Galsgaard, K., \& Priest, E. R. 2006, in preparation

Hesse, M., Forbes, T. G., \& Birn, J. 2005, ApJ, 631, 1227

Linton, M. G., \& Priest, E. R. 2003, ApJ, 595, 1259

Longcope, D. W. 1998, ApJ, 507, 650
Mellor, C., Gerrard, C. L., Galsgaard, K., Hood, A. W., \& Priest, E. R. 2005, Sol. Phys., 227, 39

Mikić, Z., Schnack, D. D., \& van Hoven, G. 1989, ApJ, 338, 1148

Milano, J. M., Dmitruk, P., Mandrini, C. H., Gómez, D. O., \& Démoulin, P. 1999, ApJ, 521,

$$
889
$$

Nordlund, Å., \& Galsgaard, K. 1997, A 3D MHD code for Parallel Computers, Technical report, Astronomical Observatory, Copenhagen University

Parker, E. N. 1972, ApJ, 174, 499

Parnell, C. E. 2001, Sol. Phys., 200, 23

Parnell, C. E. 2002, MNRAS, 335, 389

Parnell, C. E. 2002, ESA-SP, 505, 231

Parnell, C. E., \& Galsgaard, K. 2004, A\&A, 428, 595

Pevtsov, A. A., \& Acton, L. W. 2001, ApJ, 554, 416

Pontin, D. I., Galsgaard, K., Hornig, G., \& Priest, E. R. 2005, Phys. Plasmas, 12, 52307

Priest, E. R., \& Démoulin, P. 1995, J. Geophys. Res., 100 A12, 23443

Priest, E. R., Heyvaerts, J. F., \& Title, A. M. 2002, ApJ, 576, 533

Priest, E. R., Longcope, D. W., \& Heyvaerts, J. F. 2005, ApJ, 624, 1057

Priest, E. R., Hornig, G., \& Pontin, D. I. 2003, JGR, 108, 1285

Schrijver, C. J., \& Zwaan, C. 2000, Solar and Stellar Magnetic Activity (Cambridge: Cambridge Univ. Press)

Titov, V. S., Hornig, G., \& Démoulin, P. 2002, J. Geophy. Res., 107 A8, 3

Titov, V. S., Galsgaard, K., \& Neukirch, T. 2003, ApJ, 582, 1172

Van Ballegooijen 1986, ApJ, ApJ, 311, 1001 